\title{
The merger of vertically offset quasi-geostrophic vortices
}

\author{
By J. N. REINAUD AND D. G. DRITSCHEL \\ Mathematical Institute, University of St Andrews, North Haugh, St Andrews, KY16 9SS, Scotland
}

(Received 7 February 2002 and in revised form 16 May 2002)

We examine the critical merging distance between two equal-volume, equal-potentialvorticity quasi-geostrophic vortices. We focus on how this distance depends on the vertical offset between the two vortices, each having a unit mean height-to-width aspect ratio. The vertical direction is special in the quasi-geostrophic model (used to capture the leading-order dynamical features of stably stratified and rapidly rotating geophysical flows) since vertical advection is absent. Nevertheless vortex merger may still occur by horizontal advection.

In this paper, we first investigate the equilibrium states for the two vortices as a function of their vertical and horizontal separation. We examine their basic properties together with their linear stability. These findings are next compared to numerical simulations of the nonlinear evolution of two spheres of potential vorticity. Three different regimes of interaction are identified, depending on the vertical offset. For a small offset, the interaction differs little from the case when the two vortices are horizontally aligned. On the other hand, when the vertical offset is comparable to the mean vortex radius, strong interaction occurs for greater horizontal gaps than in the horizontally aligned case, and therefore at significantly greater full separation distances. This perhaps surprising result is consistent with the linear stability analysis and appears to be a consequence of the anisotropy of the quasi-geostrophic equations. Finally, for large vertical offsets, vortex merger results in the formation of a metastable tilted dumbbell vortex.

\section{Introduction}

Stably stratified, rapidly rotating turbulence, as commonly found in the atmosphere and the oceans, is largely governed by the interaction between coherent vortical structures (see for example McWilliams 1990; Dritschel \& de la Torre Juárez 1999; Koudella, Dritschel \& McMullan 2002; Reinaud, Dritschel \& Koudella 2003). Some aspects of the behaviour of these complex flows may be understood by studying how vortices interact. Indeed, it is principally through such interactions that these flows evolve. Similar statements, previously made in the context of two-dimensional turbulence, led numerous authors to focus on one aspect of vortex interactions: vortex merger, see Dritschel (1995) and references therein. Vortex merger has been thought to be responsible for the physical or vortical-space 'inverse energy cascade', i.e. energy flowing from small scales to large ones, as commonly observed in the spectral space statistics. One of the best-known results is that two identical uniform-vorticity circular vortices will merge if their separation distance is less than 3.3 times their initial radius (see Waugh 1992 and references therein).

Vortex merger has since been examined in more realistic, albeit more complicated 
geophysical flow models, most commonly in the 'quasi-geostrophic' model (Polvani, Zabusky \& Flierl 1989; Verron, Hopfinger \& McWilliams 1990; von Hardenberg et al. 2000; Dritschel 2002). The quasi-geostrophic (QG) model is based on approximate hydrostatic and geostrophic balance, yet contains the main vortex-dynamical features of stably stratified and rapidly rotating flows. Under the QG approximation and neglecting diabatic and frictional effects, such flows are fully described by the spatial distribution of the materially conserved potential vorticity, hereinafter referred to as PV, from which the velocity field is obtained by a simple linear inversion relation. Notably the vertical component of the velocity field is negligible, rendering the motion 'layerwise two-dimensional'.

Early computations examined vortex interactions in two-layer QG flows (Polvani et al. 1989; Verron et al. 1990). However, two-layer flows cannot account for important dynamical features found in continuously stratified QG flows, in particular vortex merger (Dritschel 2002). More recently, simulations of vortex merger in continuously stratified flows have been carried out by von Hardenberg et al. (2000) and Dritschel (2002). These authors considered the interaction of two identical PV ellipsoids, centred initially on the same vertical plane. As in the original two-dimensional problem, these authors sought to determine the critical horizontal separation distance below which the two vortices merge or otherwise strongly interact. Now however the problem depends on the initial vortex height-to-width aspect ratio. The limit where this aspect ratio is taken to infinity was thought to be relevant to the two-dimensional merger problem, according to the two-layer results (Verron et al. 1990; von Hardenberg et al. 2000). However, Dritschel (2002) showed that no such two-dimensional limit exists for continuously stratified unbounded flows. Instead, the vortices break down baroclinically, or three-dimensionally, with merger occurring over a relatively small vertical range between the two original vortices. This result appears to be related to the 'tall-column' instability (Dritschel \& de la Torre Juárez 1996), which results in the breakdown of vortex columns of sufficiently great height-to-width aspect ratio.

These studies were restricted to horizontally aligned vortices. In complex flows, such as turbulence, the location of vortices is likely to be random and therefore little can be drawn from these studies about vortex interactions in general.

In this paper, we go a step further by additionally considering a vertical offset between two identical uniform-PV vortices. We aim to determine the critical merging distance as a function of both the horizontal and vertical offsets. Note that, in the QG model, the flow is constrained to horizontal layerwise motion, and thus only the $\mathrm{PV}$ at equal height can merge; vortices that are vertically separated (do not share any common vertical level) cannot merge. A vertical offset between the two vortices is therefore expected to be significant.

Here we take two approaches. In the first, co-rotating equilibrium states are generated numerically. These states are then analysed for their linear stability. Instability is here associated with merger or a strong interaction. In the second approach, we simply take two identical vortex spheres, which are not in mutual equilibrium, and study their nonlinear interaction by direct numerical simulation.

Recently, Reinaud et al. (2002) showed that vortices with a height-to-width aspect ratio slightly less than unity (around 0.8 ) are statistically most likely to be found in quasi-geostrophic turbulence, in a coordinate system stretched in the vertical by the ratio of the buoyancy frequency to the Coriolis frequency. For the sake of simplicity, we limit our investigation to vortices that have a unit height-to-width aspect ratio. Moreover we will focus on equal-volume vortices. The full vortex interaction problem 
depends on the vortex aspect ratios, volume ratio, and the horizontal and vertical separation - five parameters even for equal, uniform PV!

The paper is organized as follows. Section 2 briefly reviews the quasi-geostrophic model. In $\S 3$, the equilibrium states for two unit height-to-width aspect ratio vortices are discussed, including the method used to generate them. Section 4 presents the linear stability analysis of these vortices, and $\S 5$ addresses the numerical simulation results for the nonlinear interaction of two spheres of PV. Our conclusions are given in $\S 6$.

\section{The quasi-geostrophic equations}

The quasi-geostrophic equations are obtained from an asymptotic expansion of Euler's equations for $\epsilon=H / L \ll 1$, where $H$ and $L$ are the characteristic vertical and horizontal length scales, and for $F r^{2} \ll R o \ll 1$ where $F r$ and $R o$ are respectively the Froude and Rossby numbers, see e.g. Gill (1982) for a complete discussion. We denote the Coriolis frequency by $f$, and the buoyancy frequency by $N$. Following von Hardenberg et al. (2000), Dritschel (2002), and many previous studies, we take both $f$ and $N$ to be constant.

In rescaled coordinates in which the vertical coordinate is stretched by the factor $N / f$, the governing equations read

$$
\begin{gathered}
\frac{\mathrm{D} q}{\mathrm{D} t}=0, \\
\Delta \psi=q, \\
u=-\frac{\partial \psi}{\partial y}, \quad v=\frac{\partial \psi}{\partial x},
\end{gathered}
$$

where $q(x, y, z, t)$ is the $\mathrm{PV}, \psi$ is the streamfunction and $(u, v)$ is the horizontal velocity. In (1), $\mathrm{D} / \mathrm{D} t=\partial / \partial t+u \partial / \partial x+v \partial / \partial y$ stands the material derivative, and $\Delta$ is the three-dimensional Laplace operator. Here, in absence of diabatic and dissipative effects, PV is materially conserved as stated by (1). Moreover, PV is constrained to move in horizontal layers (i.e. tangent to stratification surfaces).

In this work, we consider vortices having uniform PV. In this case, the dynamics of the flow can be fully described through knowledge of the vortex boundary shape. This is arguably the simplest context for the study of vortex merger.

\section{The steady states}

We seek the equilibrium shapes of two steadily rotating vortices having equal uniform PV and equal volume and having a prescribed vertical and horizontal separation. The vortices are stationary in a uniformly rotating reference frame, rotating at an angular velocity $\Omega$. The numerical method used here was originated by Pierrehumbert (1980) and was further developed by Dritschel (1985) and Dritschel (1995) in planar two-dimensional flows and by Polvani \& Dritschel (1993) for two-dimensional flows on the surface of a sphere. The numerical procedure used here for the three-dimensional QG problem derives straightforwardly from the latter work and is outlined in the Appendix.

Several basic quantities are calculated for the converged solutions. The first is the angular impulse of the flow. In QG flows, the angular impulse (a conserved quantity) 


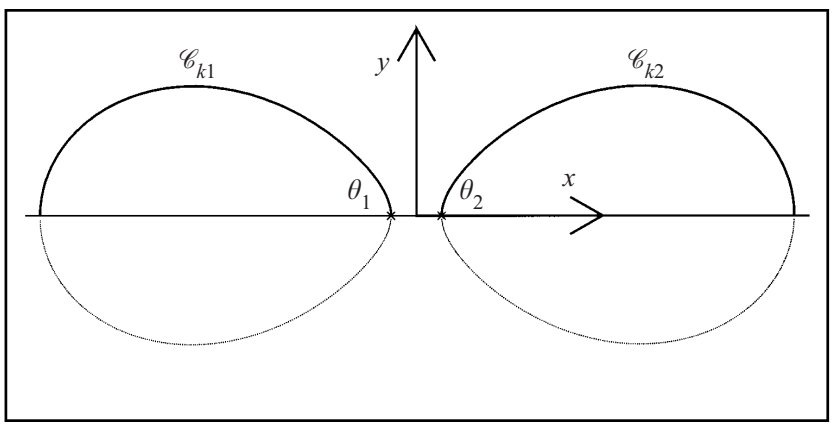

FIGURE 1. Top view of the mid-layer in each of the two vortices and definition of parameters used to define the steady states. $\theta$ is the angle with respect to the positive $x$-axis.

is defined by

$$
I=\iiint \rho^{2} q \mathrm{~d} v
$$

and may be reduced to contour integrals. The second is the excess energy defined by

$$
T=-\frac{1}{2} \iiint q \psi \mathrm{d} v .
$$

This is also conserved and may be reduced to contour integrals. These two quantities are important in the analogous two-dimensional study (Dritschel 1995), and turn out to be important here as well.

\subsection{Small to moderate vertical offsets}

The steady states obtained for the two equal-volume, equal-uniform-PV vortices are now presented. For the computations, each vortex is discretized by 25 horizontal contours and each contour by 238 nodes. The total volume of PV is the same for all the simulations and is set to $4 \pi / 3$. The PV itself within each contour is set to $2 \pi$. The contour areas $\mathscr{A}_{k}$ are set by the condition that the vortices have a unit height-to-width aspect ratio; that is, the areas correspond to those of a perfectly spherical vortex spanning the same height range. Note that an infinite isolated column of PV has a rotation period of $4 \pi /|q|$ while a sphere has a period of $6 \pi /|q|$. Steady solutions for a large range of both horizontal $\delta x$ and vertical $\delta z$ offsets, defined below, are investigated.

The centroids of the vortices lie in the plane $y=0$ and the vortices are symmetric across the plane $y=0$ (see figure 1). Consequently, iterative corrections to find the steady state are only computed on half of each contour. For each vertical offset between the vortex centroids $\delta z$, the calculation is initialized using two well-separated identical spheres of radius $R \equiv(3 V / 4 \pi)^{1 / 3}=2^{-1 / 3}$ where $V=2 \pi / 3$. This is justified by the fact that an isolated radial distribution of PV is indeed a steady state. The first guess for the background rotation rate is given by the equivalent point-vortex formula $\Omega_{p}=2 \pi /\left(3\left(\delta x^{2}+\delta z^{2}\right)^{3 / 2}\right)$ obtained for two point vortices of strength $q V=4 \pi^{2} / 3$. The initial horizontal offset between the vortices $\delta x$ is set to 3.5 . When a steady state is found, the horizontal separation distance between the two vortices is reduced and the numerical procedure is resumed for this new separation distance. The new first guess for this configuration is obtained by linear extrapolation from the previous two steady states. This decreases the number of iterations necessary to reach the next 


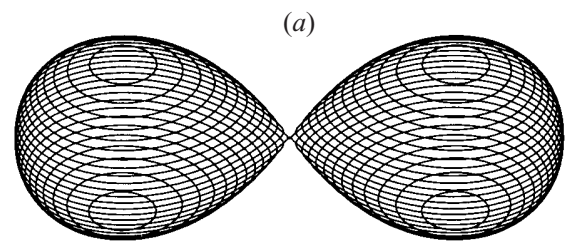

(c)

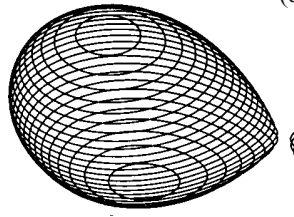

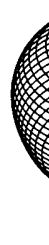
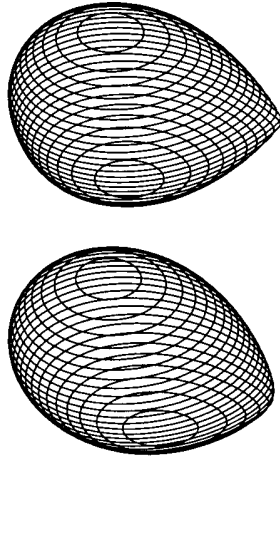

(b)

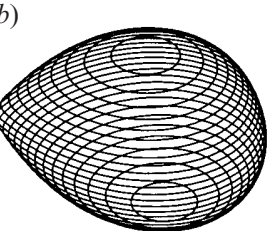

(d)
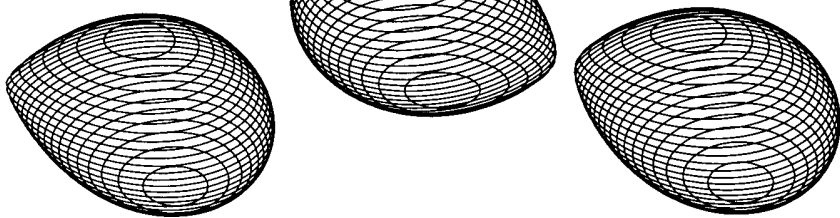

FIGURE 2. Illustration of the steady states with $(a) \delta z / R=0$ and $\delta x=2.286,(b) \delta z / R=0.4$ (5 layers) and $\delta x=2.277,(c) \delta z / R=0.8$ (10 layers) and $\delta x=2.243$ and $(d) \delta z / R=1.2$ (15 layers) and $\delta x=2.164$. The contours are viewed orthographically, at an angle of $45^{\circ}$ from the vertical.

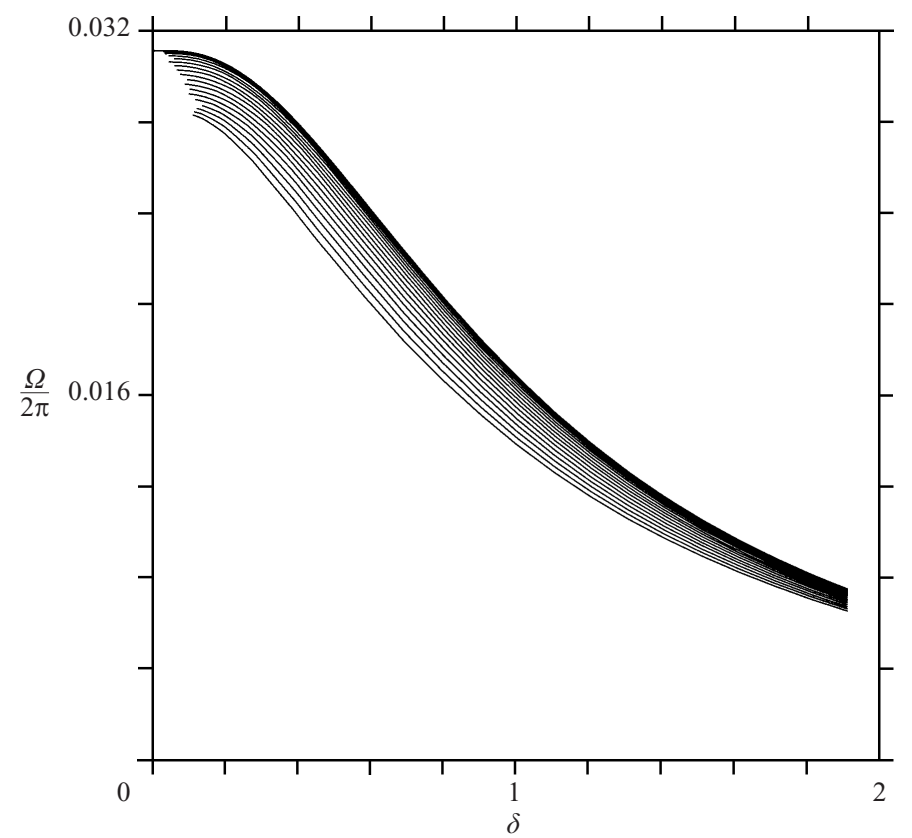

FigURE 3. Scaled background rotation $\Omega /(2 \pi)$ versus the horizontal gap $\delta$. In this and the figures 4-8 (except where noted), the upper curve corresponds to $\delta z / R=0$ and the bottom curve to $\delta z / R=1.44$, while the increment in $\delta z / R$ between the curves is 0.08 .

steady state. Note that the iterative procedure corrects the value of $\delta x$. Hereinafter, $\delta x$ will refer to the converged value of the horizontal offset.

Families of steady states (spanned by $\delta x$ ) have been studied for 19 different vertical offsets $\delta z$ in increments of the layer thickness starting from $\delta z=0$ and ending at $\delta z=18 \Delta z$ (i.e. $\delta z / R=1.44$ ). Recall that each vortex is spanned by 25 layers. Note here that, unlike the horizontal offset, $\delta z$ is unchanged by the iterative procedure.

The steady states with the smallest horizontal offset $\delta x$ are illustrated in figure 2 for four different vertical offsets $(\delta z / R=0,0.4,0.8$ and 1.2). Note that the vortices 


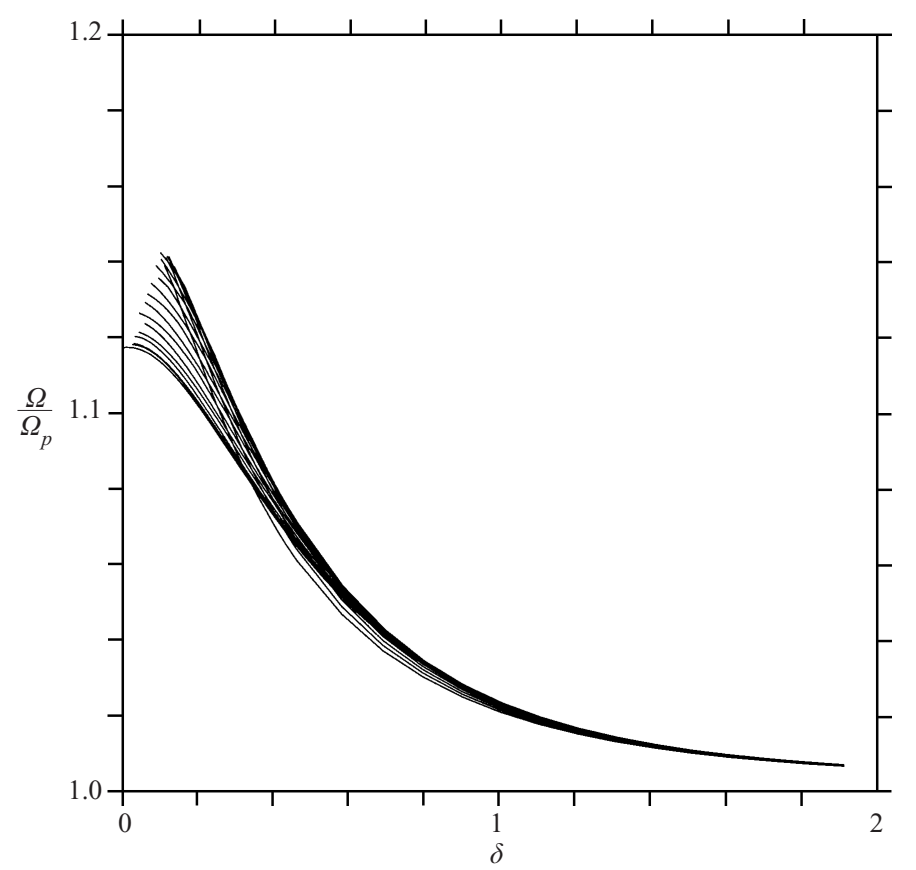

FIGURE 4. Rotation ratio $\Omega / \Omega_{p}$ versus the horizontal gap $\delta$, for $0 \leqslant \delta z / R \leqslant 1.44$. Here, the curves for different values of $\delta z / R$ overlap one another.

exhibit sharp inner corners. The contour shapes are similar to the ones that have been obtained for two-dimensional co-rotating vortices, see e.g. Dritschel (1995), and are characteristic of unstable configurations. Figure 3 gives the scaled rotation $\Omega /(2 \pi)$ of the two vortices as a function of the horizontal gap $\delta$, that is the horizontal distance between the innermost edges of the two vortices. In this and the following five figures, the upper curve corresponds to $\delta z / R=0$ and the bottom curve to $\delta z / R=1.44$, while the increment in $\delta z / R$ between two curves is 0.08 . The rotation rate $\Omega$ increases as the horizontal gap decreases and the interaction between the two vortices becomes stronger. Likewise, $\Omega$ decreases as the vertical offset $\delta z$ increases, consistent with the point-vortex formula $\Omega_{p}=2 \pi /\left(3\left(\delta x^{2}+\delta z^{2}\right)^{3 / 2}\right)$. In fact, $\Omega_{p}$ is a good approximation to $\Omega$, as shown in figure 4 , which plots $\Omega / \Omega_{p}$ versus $\delta$ for various $\delta z$. It is remarkable that even for almost touching vortices, $\Omega_{p}$ is accurate to within $10 \%$ of the true value. This demonstrates that the point-vortex model provides an accurate leading-order estimate of the vortex interaction.

The dependence of the angular impulse $I$ on the horizontal gap $\delta$ is shown in figure 5 for the 19 families of steady state. It first decreases with $\delta$ and reaches a minimum value $I_{m}$ at a critical gap $\delta=\delta_{m}$, and thereafter increases. The location of this minimum value is indicated on the curves by a triangle (see also the close-up provided in figure 6). Such a minimum in $I$ as a function of $\delta$ also occurs in the analogous two-dimensional problem, cf. Dritschel (1995). Moreover, Dritschel (1995) found that this minimum occurs at the margin of stability for like-signed vortices (states with $\delta<\delta_{m}$ are unstable). As shown in the next section, this correspondence between the minimum of $I$ and linear stability is found here as well. As a function of the vertical gap $\delta z$, the angular impulse decreases as $\delta z$ increases. Note, from (4), that the angular impulse is not an explicit function of the vertical offset $\delta z$, so the 


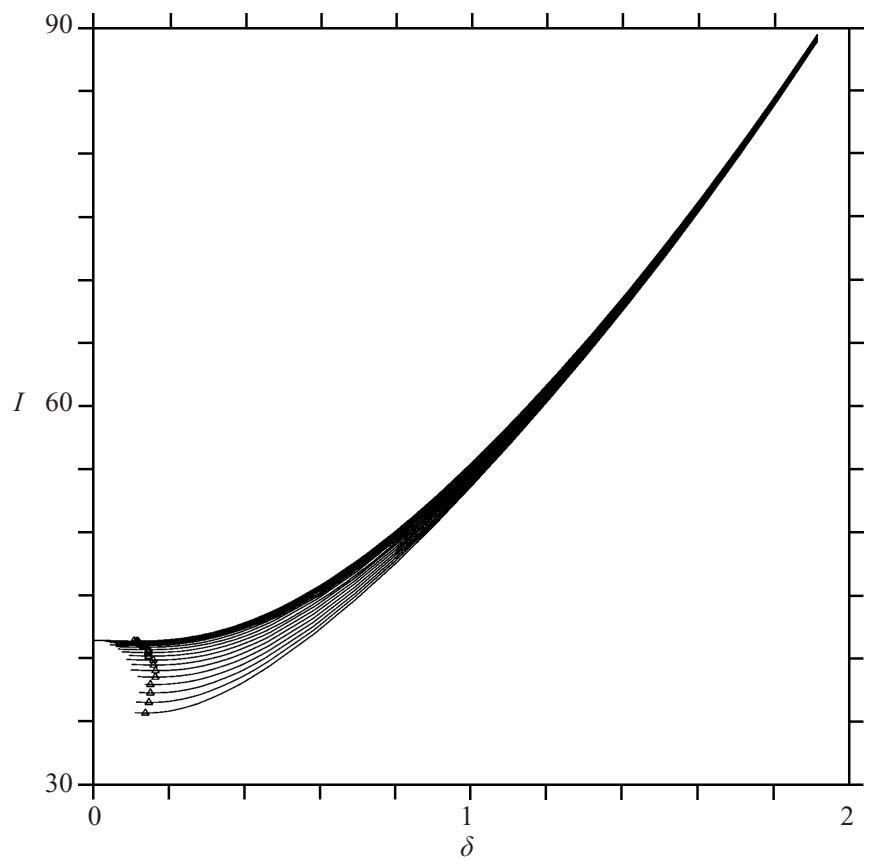

FIGURE 5. Angular impulse $I$ versus the horizontal gap $\delta$. The triangles indicate the location of the minimum $I_{m}$. See the caption of figure 3 for details of the curves plotted.

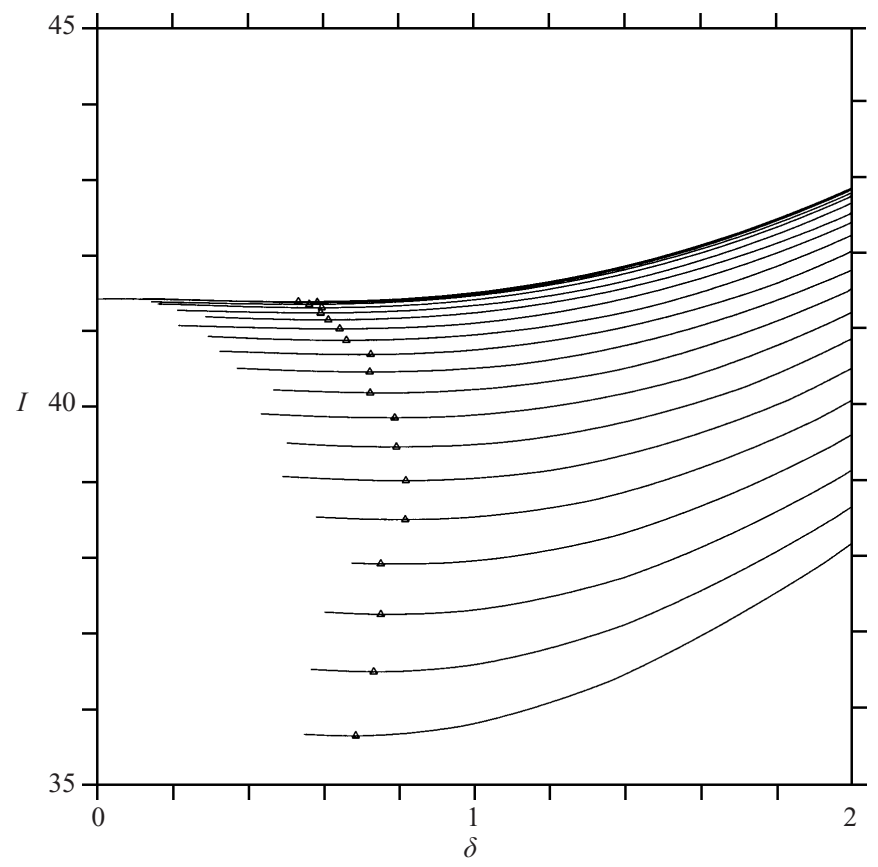

Figure 6. Close-up: Angular impulse $I$ versus the horizontal gap $\delta$. The triangles indicate the location of the minimum $I_{m}$. 


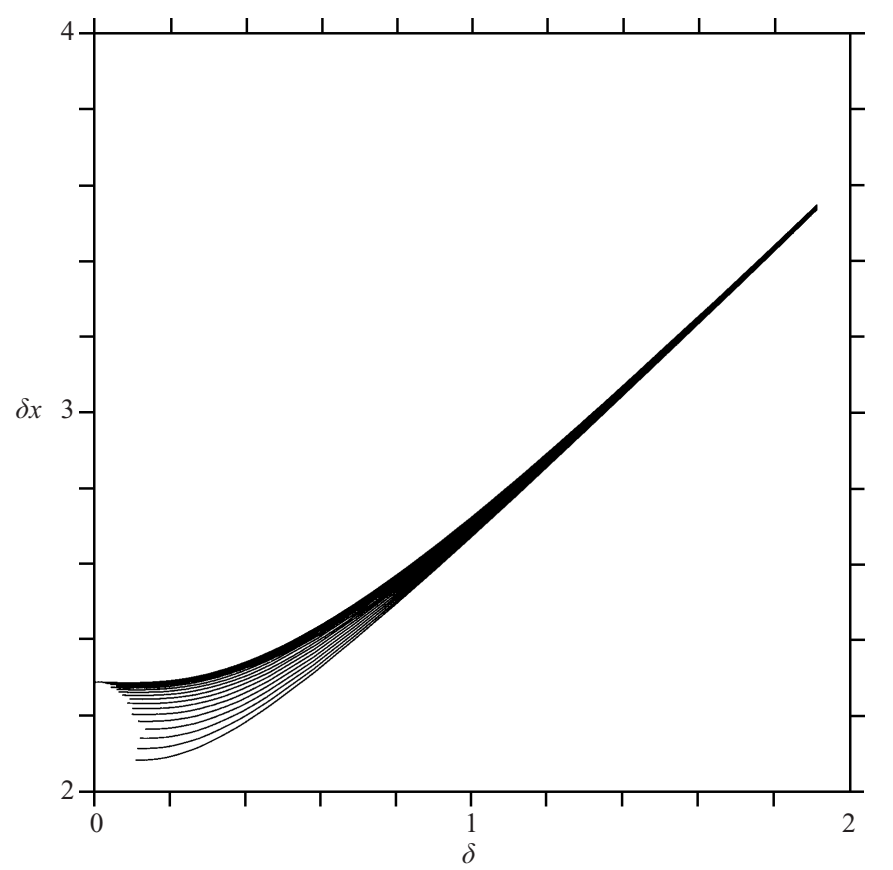

FIGURE 7. Horizontal centroid separation distance $\delta x$ versus the horizontal gap $\delta$.

observed variation is due to the changing shape of the vortices with $\delta z$. This implies that, for a given horizontal gap $\delta$, the horizontal offset between the PV-centroids $\delta x$ decreases when the vertical offset $\delta z$ increases.

We next present the horizontal distance between the vortex centroids - the offset $\delta x$ - as a function of the horizontal gap $\delta$ in figure 7. This confirms that, as suggested by the dependence of $I$ on $\delta, \delta x$ decreases as $\delta z$ increases, for a given horizontal gap $\delta$. Moreover, $\delta x$ decreases more slowly than does $\delta$, indicating that the vortices become more deformed as they approach each other. Note also that the distance between the inner edge and the centroid increases with decreasing $\delta$.

Finally, figure 8 shows the dependence of the excess energy $T$ on the horizontal gap $\delta$ for various vertical offsets $\delta z$. The excess energy first increases as the gap $\delta$ decreases, reaches a maximum $T=T_{M}$ at $\delta=\delta_{m}$, then decreases for $\delta<\delta_{m}$. The maximum (indicated by triangles in the figure) occurs at the same point as where the angular impulse $I$ reaches a minimum. This also occurs in the analogous twodimensional problem (Dritschel 1995). For fixed $\delta$, the excess energy decreases with increasing $\delta z$, indicating a weakening of the vortex interaction energy.

\subsection{Higher vertical offsets}

In the previous subsection, we restricted attention to steady states having small to moderate vertical offsets $0<\delta z / R<1.44$. We have also found families of steady states for larger vertical offsets. However, for these families, we were unable to reach the margin of stability, despite trying a number of modifications to our iterative solution method. When the vortices approach one another and begin to exhibit sharp inner edges, the numerical procedure becomes unstable and eventually diverges. Similar numerical difficulties were encountered in Polvani \& Dritschel (1993) and in Dritschel (1995) in the two-dimensional context. We believe that these numerical instabilities 


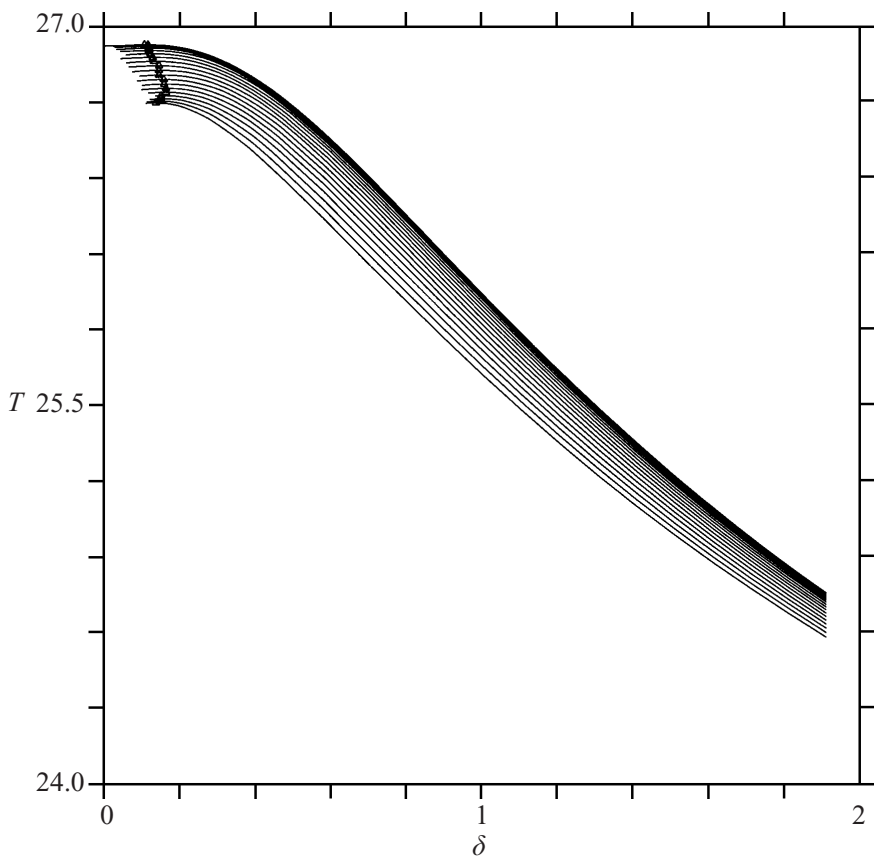

FIGURE 8. Excess energy $T$ versus the horizontal gap $\delta$. The triangles indicate the location of the maximum $T_{M}$.

are associated with the proximity of another family of steady states, in this case a single compound vortex.

Evidence for this is provided next. We took the last steady state (with the smallest $\delta$ for $\delta z=19 \Delta z$, corresponding to $\delta z / R=1.52$ ), and pushed each vortex towards the other slightly to initialize a nonlinear numerical simulation of the flow evolution. This steady state is characterized by a background rotation rate $\Omega=0.028007$, an angular impulse $I=34.698$, an excess energy $T=26.695$ and a horizontal offset $\delta x=2.0489$. The horizontal gap $\delta$ is just $3.3 \%$ of the horizontal span of the pair of vortices, measured here as the distance between their two outer edges. This steady state has been found to be linearly stable. To initialize the numerical simulation, we reduced $\delta x$ by just 0.0002 . The simulation was performed using the Contour Surgery (CS) algorithm, (see Dritschel 2002 and references therein) from which our steadystate iterative procedure has been developed. The nonlinear evolution of the vortices is illustrated in figure 9. After a moderately long period of nearly steady motion, a compound state forms when the two vortices bridge at $t=26.5$. This compound vortex persists for a long period of time but eventually destabilizes and separates into two asymmetric vortices at $t=131$. After a new attempt to form a compound vortex at $t=133$, the vortex separates at $t=136$ and has not reconnected at $t=500$, the end of the simulation. The final state consists of an asymmetric pair of vortices with a volume ratio of 1.72 . This result suggests that the last steady state obtained is close to a new branch of singly connected equilibria, which however are unstable, at least near the point of bifurcation into a pair of vortices.

Additional simulations starting with two spheres having large vertical offsets are presented in $\S 5$ and confirm the creation of meta-stable compound states in this regime. 


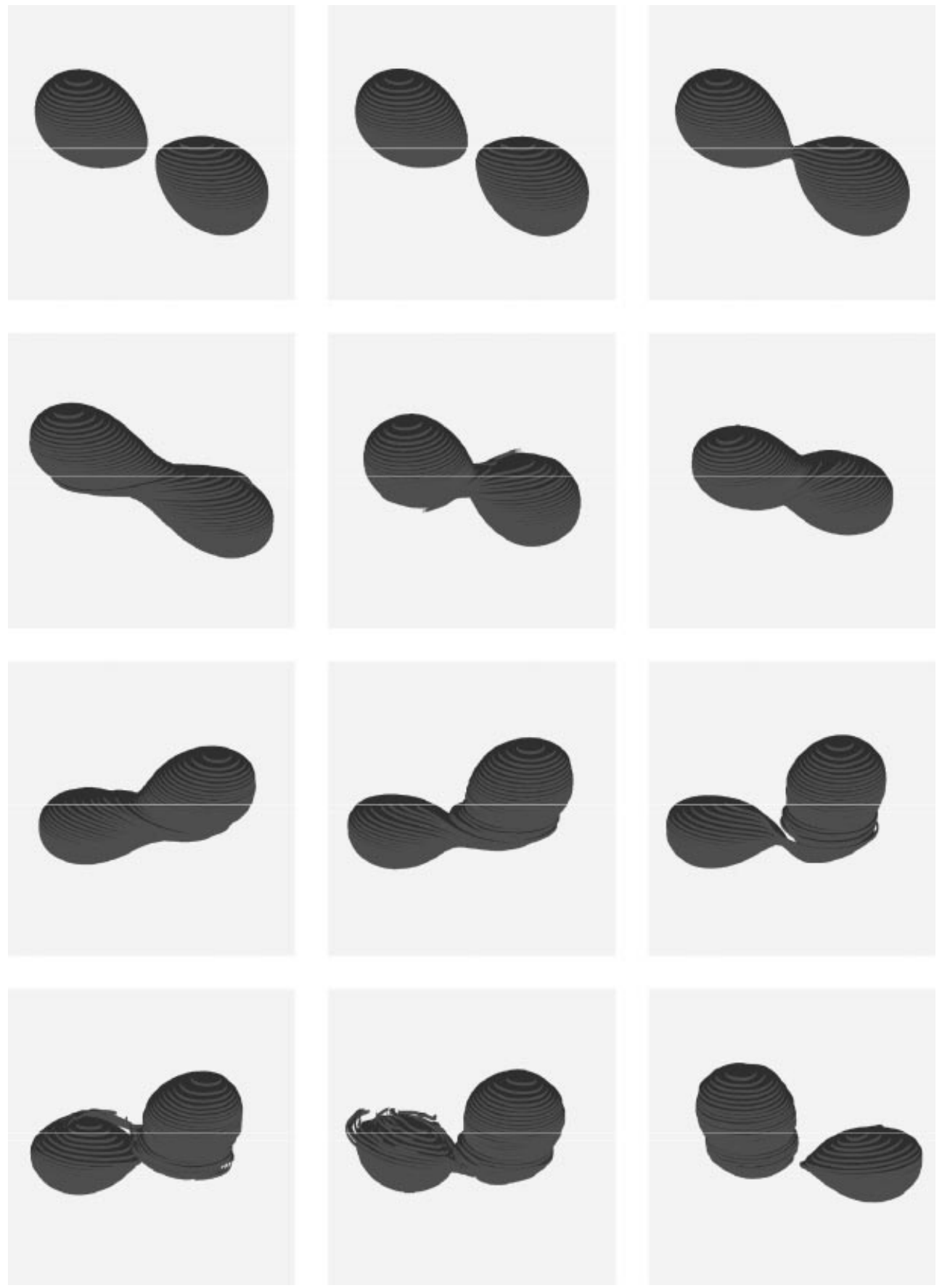

FIGURE 9. Illustration of the nonlinear evolution of the last steady vortices obtained for a vertical offset of 19 layers $(\delta z / R=1.52)$ after being slightly pushed together. Times displayed (left to right, then top to bottom) are $t=0,20,27,35,47,56,115,120,123,131,135$ and 230. The evolution was computed using the CS algorithm with time step $\Delta t=0.025$, dimensionless node separation parameter $\mu=0.150$, and large-scale length $L=1.0$. 


\section{Linear stability}

We now present the linear stability of the steady states obtained in the previous section. Our principal goal here is to determine the horizontal gap $\delta_{m}$ below which unstable modes exist for various vertical offsets between the two vortices. This gap $\delta_{m}$ gives the limit below which inelastic interactions may be expected to occur between the vortices.

\subsection{Formulation}

The formulation of the numerical approach is first briefly described. It is based upon the analysis of small deformations to the contours defining the boundaries of the vortices, as proposed in two-dimensional flows by Dritschel (1995) and Polvani \& Dritschel (1993). The perturbed boundary of a given PV-contour $\mathscr{C}_{k}$ is written as

$$
\boldsymbol{\rho}_{k}\left(\phi_{k}, t\right)=\boldsymbol{\rho}_{e, k}\left(\phi_{k}\right)+\gamma_{k}\left(\phi_{k}, t\right) \frac{\left\{\mathrm{d} y_{e, k} / \mathrm{d} \phi_{k}-\mathrm{d} x_{e, k} / \mathrm{d} \phi_{k}\right\}}{\left(\mathrm{d} x_{e, k} / \mathrm{d} \phi_{k}\right)^{2}+\left(\mathrm{d} y_{e, k} / \mathrm{d} \phi_{k}\right)^{2}},
$$

where $\phi_{k}$ represents here the 'travel time coordinate', i.e. a quantity proportional to the time that a fluid particle takes to travel along the contour $\mathscr{C}_{k}, \boldsymbol{\rho}_{e, k} \equiv\left(x_{e, k}, y_{e, k}\right)$ is the horizontal position vector describing the equilibrium shape, and $\gamma_{k}$ is the (infinitesimally small) perturbation in the normal direction to the contour $\mathscr{C}_{k}$. The angular coordinate $\phi_{k}$ is scaled so that $0<\phi_{k}<2 \pi$ maps each contour. Note that $\gamma_{k}\left(\phi_{k}, t\right)$ has the dimensions of area. The perturbation $\gamma_{k}$ is governed, at first order, by the equation

$$
\frac{\partial \gamma_{k}}{\partial t}+\Omega_{e, k} \frac{\partial \gamma_{k}}{\partial \phi_{k}}=-\sum_{l=1}^{n_{c}} \Delta q_{l} \frac{\partial}{\partial \phi_{k}} \oint_{\mathscr{C}_{l}} \gamma_{l} G_{k, l}(\varrho) \mathrm{d} \phi_{l}^{\prime},
$$

where $G_{k, l}(\varrho)$ is the Green function giving the velocity induced in the layer containing the contour $\mathscr{C}_{k}$ by the PV within the contour $\mathscr{C}_{l}, \Delta q_{l}$ is the PV jump across $\mathscr{C}_{l}$, $\Omega_{e, k}$ is the constant rotation rate of fluid particles along $\mathscr{C}_{k}, \varrho=\left|\boldsymbol{\rho}_{e, k}\left(\phi_{k}\right)-\boldsymbol{\rho}_{e, l}\left(\phi_{l}^{\prime}\right)\right|$ denotes the horizontal distance between the contour points, and $n_{c}$ denotes the total number of contours. Equation (7) is the straightforward generalization of the twodimensional formula derived in Dritschel (1995) to the three-dimensional QG model (see also Dritschel \& de la Torre Juárez 1996).

The perturbation function $\gamma_{k}$ is expanded in a truncated Fourier series expansion as

$$
\gamma_{k}\left(\phi_{k}, t\right)=\mathrm{e}^{\sigma t} \sum_{m=1}^{M} A_{k, m} \cos \left(m \phi_{k}\right)+B_{k, m} \sin \left(m \phi_{k}\right) .
$$

Here, we use $M=10$ azimuthal modes (the results prove insensitive to doubling $M$, except very close to the margin of stability, as to be expected). Equation (7) can then be written as a matrix eigenvalue problem for $\sigma$. Since the matrix order is $2 M n_{c}$ by $2 M n_{c}$, there are $2 M n_{c}$ eigenvalues, $\sigma=\sigma_{j}$ (generally complex), with $j$ denoting the index of the eigenmode. (The corresponding spatial structure of this eigenmode is given by (8) with $A_{m, k}=A_{j, m, k}$ and $B_{m, k}=B_{j, m, k}$.) The equilibrium is unstable if there exists an eigenvalue $\sigma_{j}$ with a positive real part, $\sigma_{j}^{r}>0$. Otherwise it is neutrally stable (all eigenmodes have $\sigma_{j}^{r}=0$ ). Stable eigenmodes with $\sigma_{j}^{r}<0$ only occur in conjunction with unstable modes, and modes with non-zero frequency $\sigma_{j}^{i}$ always come in conjugate pairs $\left(\sigma=\sigma_{j}^{r}+\mathrm{i} \sigma_{j}^{i} \Rightarrow \sigma=\sigma_{j}^{r}-\mathrm{i} \sigma_{j}^{i}\right.$ also). This is a consequence of the Hamiltonian structure of the original problem. 

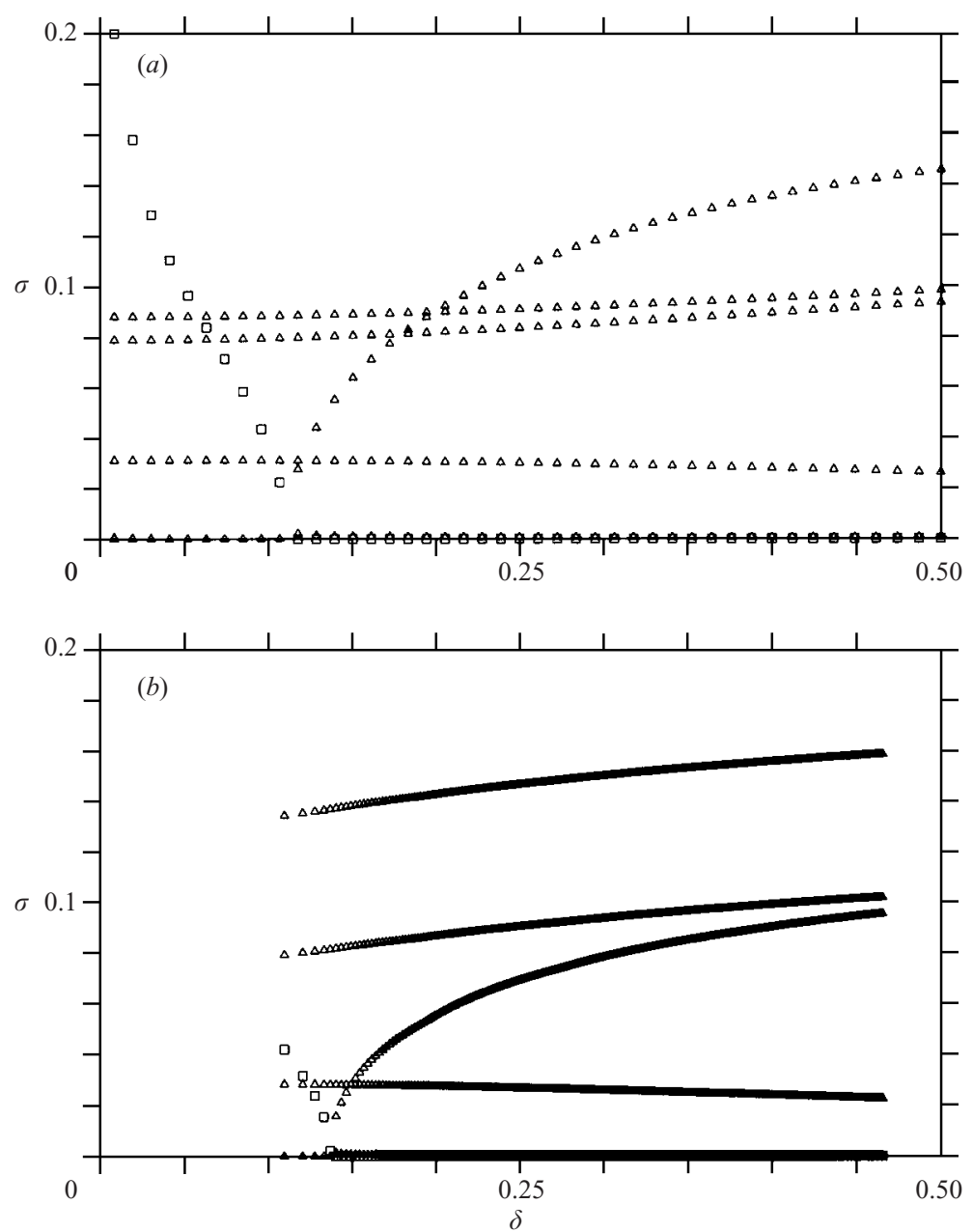

FIGURE 10. The growth rates $\sigma_{r}$ (squares) and frequencies $\sigma_{i}$ (triangles) versus the horizontal gap $\delta$ for $(a) \delta z / R=0$ and $(b) \delta z / R=1.44$ (18 layers).

\subsection{Results}

We first illustrate the behaviour of the eigenvalues $\sigma_{j}$ for two given vertical offsets, $\delta z=0$ and $\delta z=18$ layers $(\delta z / R=1.44)$, as a function of the horizontal gap $\delta$. The results are shown in figure $10(a, b)$. They are representative of all vertical offsets examined. One of the neutral eigenmodes, hereinafter referred to as the 'critical mode', has a frequency $\sigma^{i}$ which decreases more rapidly than all of the others as the gap decreases. This frequency (and its conjugate with $\sigma^{i}<0$ ) eventually collapses to zero at $\delta=\delta_{m}$ and become a stable/unstable mode pair for smaller $\delta$. This mode pair is non-propagating $\left(\sigma^{i}=0\right)$ for all $\delta<\delta_{m}$. Similar behaviour occurs in the analogous two-dimensional problem (Dritschel 1995). The margin of instability $\delta=\delta_{m}$ is found to correspond the minimum of angular impulse $I_{m}$ and the maximum of excess energy $T_{M}$ as also found in the two-dimensional problem for like-signed vortices (Dritschel 1995). Finally we note that the frequency $\sigma^{i}$ with which the neutral critical mode travels along the vortex for a fixed $\delta>\delta_{m}$ decreases with increasing vertical offset $\delta z$. 

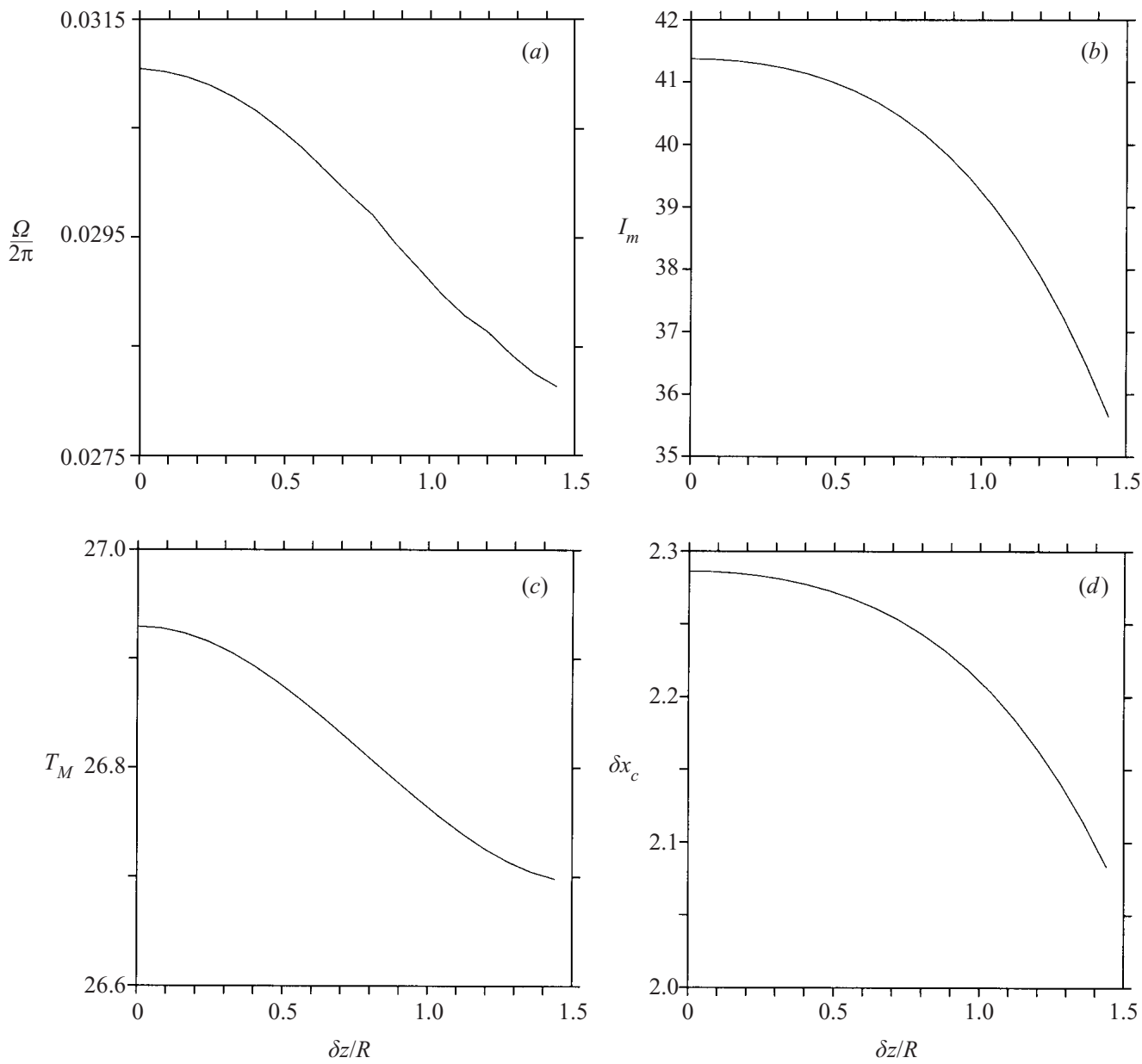

FiguRE 11. (a) Scaled rotation rate $\Omega /(2 \pi)$ at the margin of stability, $(b)$ the minimum angular impulse $I_{m},(c)$ the maximum excess energy $T_{M}$ and $(d)$ the critical horizontal centroid separation distance $\delta x_{c}$, all as functions of $\delta z / R$.

Equilibrium properties at the margin of stability are now examined. Figure 11(a-d) shows the dependence of these properties on the vertical offset $\delta z$ : in $(a)$ the 'critical' background rotation $\Omega_{c} /(2 \pi)$, in $(b)$ the minimum angular impulse $I_{m}$, in $(c)$ the maximum excess energy $T_{M}$, and in $(d)$ the critical horizontal offset $\delta x_{c}$. It is found that $\Omega_{c}, I_{m}$ and $T_{M}$ decrease as the vertical offset $\delta z$ increases. Initially, this decrease is slow, indicating that small vertical offsets only slightly modify the basic characteristics of the interaction. But while the vortex centroids are closer horizontally for larger vertical offsets, in fact the overall distance between the centroids at the margin of stability, $d_{3 D}=\left(\delta z^{2}+\delta x_{c}^{2}\right)^{1 / 2}$, increases with $\delta z$-see figure 12 . Thus, vortices offset moderately in the vertical $(0<\delta z / R<1.44)$ destabilize at greater separations than vortices with no vertical offset. At yet larger vertical offsets, we expect that this distance should eventually decrease since merging becomes increasingly difficult (indeed impossible for $\delta z / R>2$ ).

The increase in the critical separation distance $d_{3 D}$ with $\delta z$ is related to the fact that the vortices are more deformed (i.e. less spherical) when there is a vertical offset. Note first that, because of symmetry, the two vortices share the same geometric properties. 


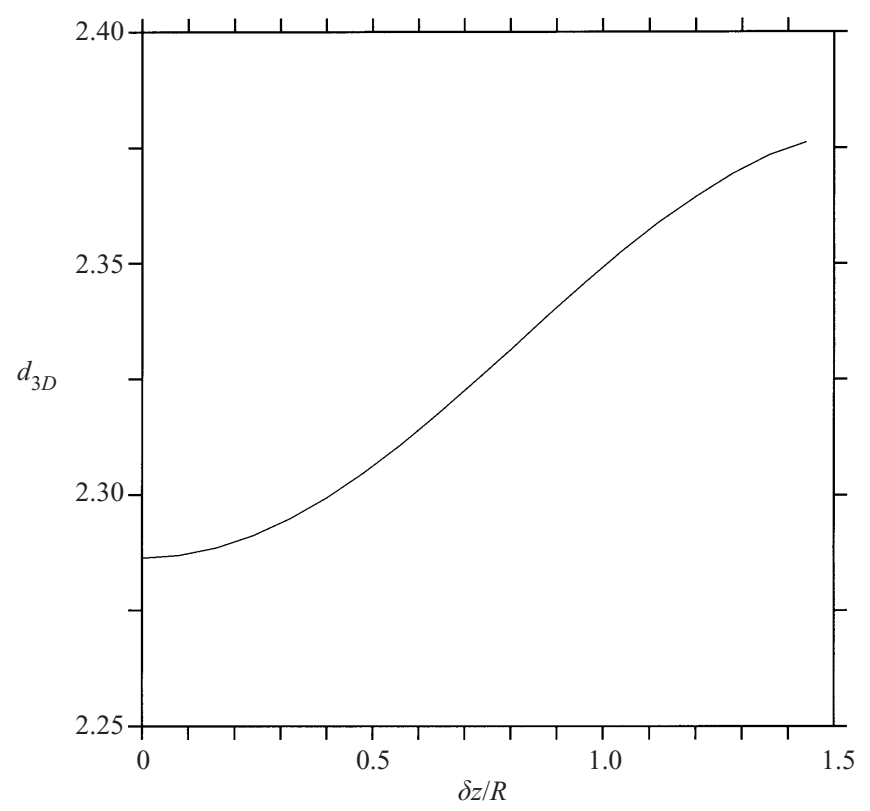

FIGURE 12. Separation distance $d_{3 D}$ at the margin of stability versus $\delta z / R$.

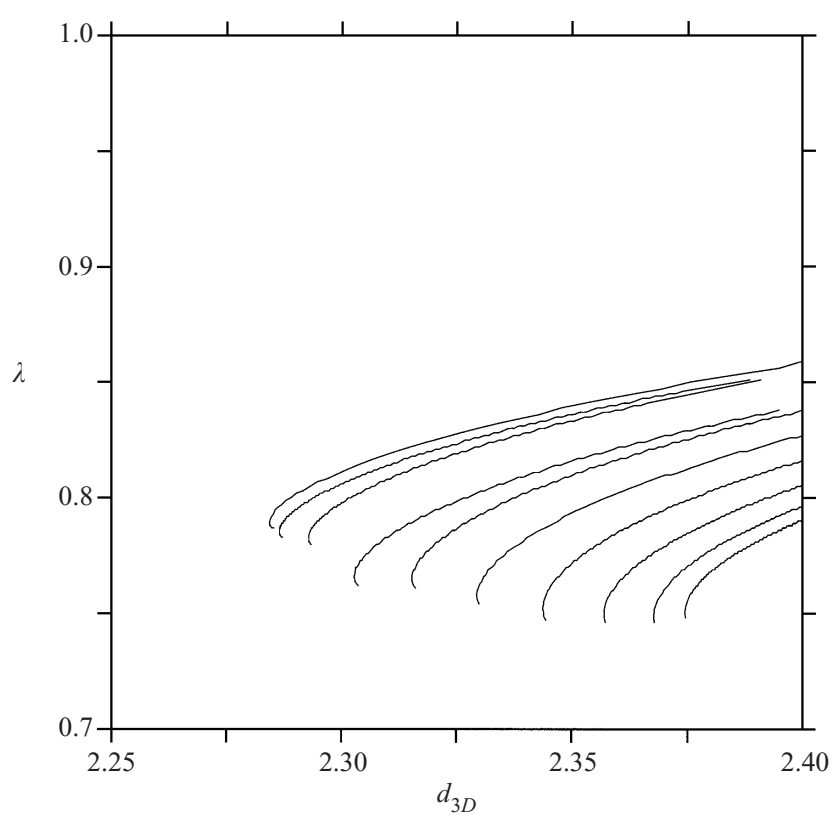

FIGURE 13. Mean aspect ratio $\sqrt{(a b)} / c$ versus $d_{3 D}$ for different vertical offsets from $\delta z / R=0$ (upper curve) to $\delta z / R=1.44$ (18 layers, bottom curve). $\delta z$ varies by $2 \Delta z=0.16 R$ between each curve.

To measure the vortex deformation, we have computed the aspect ratio $\lambda$ between an estimated 'major axis length' and a 'mean minor axis length' for one of the vortices. These lengths are obtained as follows. We first calculate the second moments of the vortex, the volume integrals of $\hat{x}^{2}, \hat{x} \hat{y}, \hat{x} \hat{z}, \hat{y}^{2}, \hat{y} \hat{z}$ and $\hat{z}^{2}$, where $\hat{\boldsymbol{x}}=(\hat{x}, \hat{y}, \hat{z}) \equiv \boldsymbol{x}-\boldsymbol{X}$, and $\boldsymbol{X}$ is the centroid of the vortex. We then associate these with an ellipsoid having 


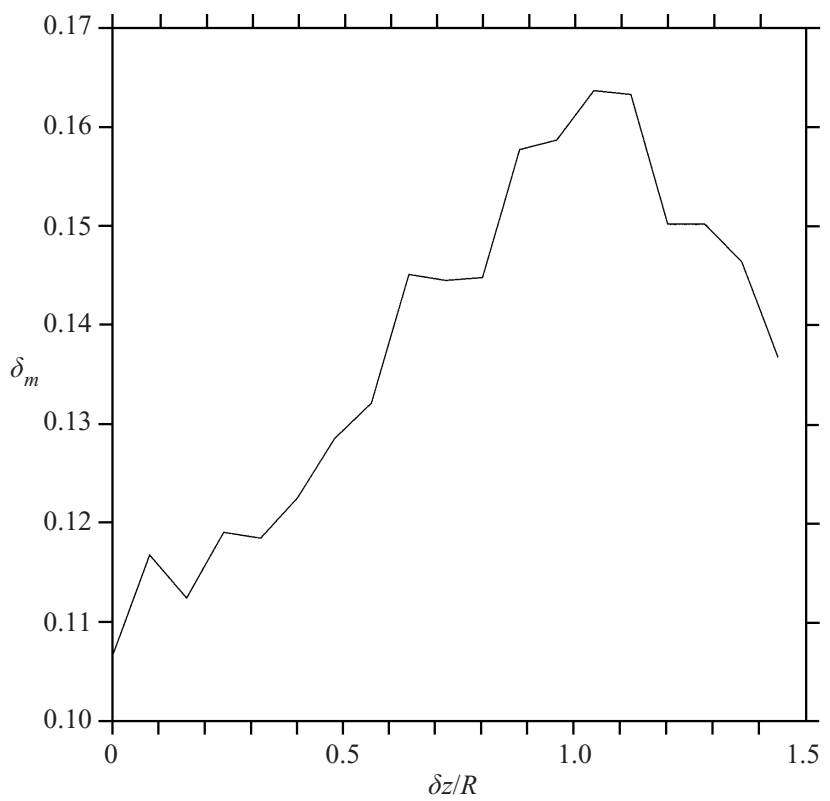

FIGURE 14. Horizontal gap $\delta$ at the margin of stability versus $\delta z / R$.

the same second moments, centroid and volume. Then $\lambda$ is calculated from the three semi-axis lengths $a \leqslant b \leqslant c$ of the ellipsoid by $\lambda=\sqrt{a b} / c$. Note that by definition $\lambda \leqslant 1$. The results are presented in figure 13 which plots $\lambda$ versus $d_{3 D}$ for various vertical offsets $\delta z$. These results demonstrate that the vortices are more deformed ( $\lambda$ is smaller) when there is a vertical offset between them. We conjecture that the greater deformation observed for vertically offset vortices is responsible for the lower frequency of the critical mode observed in figure $10(a, b)$ and the earlier onset of the instability with decreasing vortex separation $d_{3 D}$. That is, the greater vortex deformation makes it more difficult for a disturbance to propagate around the vortex boundary-this is true at least in the two-dimensional case for an elliptical vortex (cf. Dritschel 1990).

We next examine the dependence of the critical gap $\delta_{m}$ on the vertical offset $\delta z$. Figure 14 shows that the gap $\delta_{m}$ is not a monotonic function of $\delta z$. The critical gap exhibits a maximum around $\delta z / R \simeq 1.1$, indicating that vortices offset vertically by about one mean radius are the first to become unstable with decreasing horizontal gap. This observation is important for understanding the nonlinear evolution of two spheres of PV in the next section.

Finally, we examine the dependence of the growth rate $\sigma^{r}$ on both the vertical offset and the horizontal gap. Contours of $\sigma^{r}$ in the plane $(\delta z / R, \delta)$ are presented in figure 15 . These have been obtained by extrapolating the analytic relation

$$
\sigma^{r} \simeq c\left(\delta_{m}-\delta\right)^{1 / 2},
$$

which approximately holds near the margin of instability. The coefficients $c$ and $\delta_{m}$ are obtained by a least-squares fit of $\left(\sigma^{r}\right)^{2}$ to a straight line in $\delta$ (there are otherwise too few points to draw the contours). Instability at the largest horizontal gap $\delta$ occurs around $\delta z / R \simeq 1.1$, as already indicated in figure 14. Note however that for $\delta$ just less than $\delta_{m}$, the growth rates increase more steeply for $\delta z / R=0$. The instability is evidently more vigorous for horizontally aligned vortices than it is for vertically offset ones. 


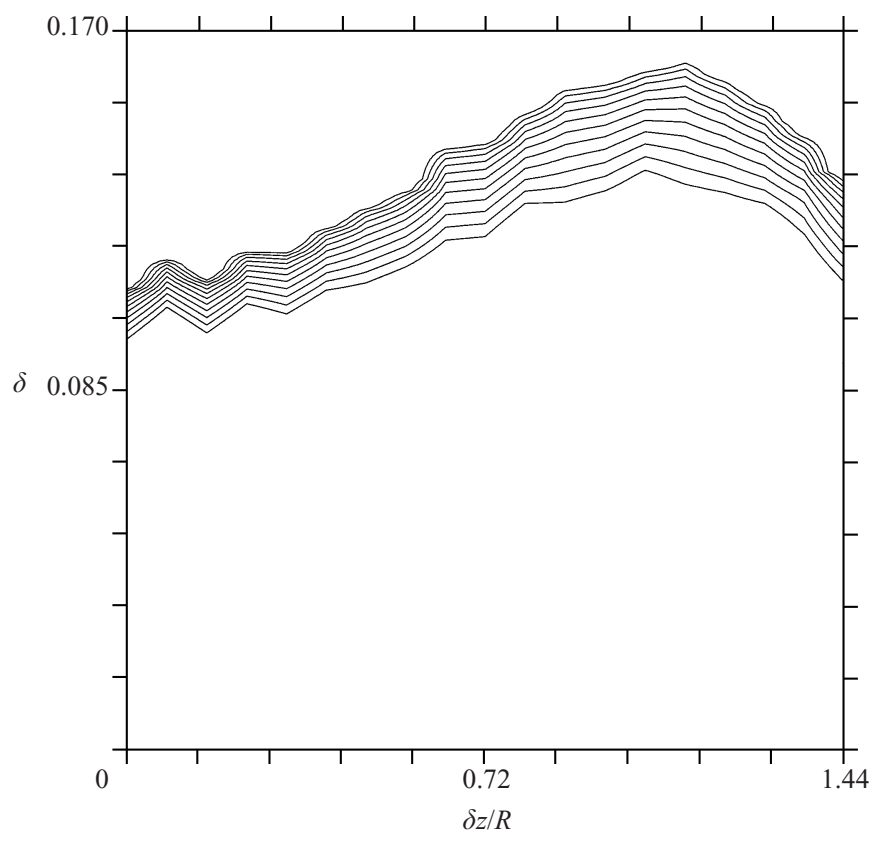

FiguRE 15. Contours of the growth rate, $0.01<\sigma^{r}<0.04$, with contour interval $\mathrm{d} \sigma_{r}=0.003$ in the plane $(\delta z / R, \delta)$. The top contour corresponds to $\sigma^{r}=0.01$ and the bottom contour to $\sigma^{r}=0.04$.

\section{Nonlinear evolution}

\subsection{Numerical procedure}

The nonlinear interaction between two identical spheres of PV is now investigated using the 'contour-advective semi-Lagrangian' (CASL) algorithm (Dritschel \& Ambaum 1997). This numerical approach, like CS, makes PV conservation explicit by tracking material contours of PV in each horizontal layer or vertical level. This approach benefits from the inherent stability of Lagrangian methods, see Cottet (1996) and Dritschel et al. (1999), and allows one to use larger time steps than Eulerian methods. The time step is taken to be inversely proportional to the maximum PV anomaly, and is typically much larger than the one required in Eulerian methods for numerical stability. Unlike CS, the CASL algorithm obtains the velocity field by inverting Poisson's equation (2) in spectral space like in the pseudo-spectral approach, and then interpolates this field at the Lagrangian contour points. Details of the algorithm can be found in Dritschel \& Ambaum (1997).

Each vortex is discretized by 40 horizontal contours of PV. Following Dritschel (2002) we ensure that the horizontal grid resolution is such that there are at least 10 grid points spanning the diameter of each vortex. The domain is triply periodic and of dimensions $\ell \times \ell \times d$, with $\ell=2 \pi$ and $d=2 \pi(R+\delta z)$. The radius of the vortices is chosen so that their outer edges touch $x= \pm 1$. This mitigates the influence of the periodic boundary conditions (see Dritschel 2002; Dritschel \& Macaskill 2000 for further remarks on the adverse effects of periodicity). The PV of both vortices is set to $Q=4 \pi$ and the time step used is $\Delta t=0.025$ (as recommended in Dritschel \& Ambaum 1997). All simulations are continued until $t=100$, which is considered the 'final' state.

The shape of the vortices is monitored as follows. We first identify the vortices present in the flow; they are defined to be contiguous regions of PV. From the 


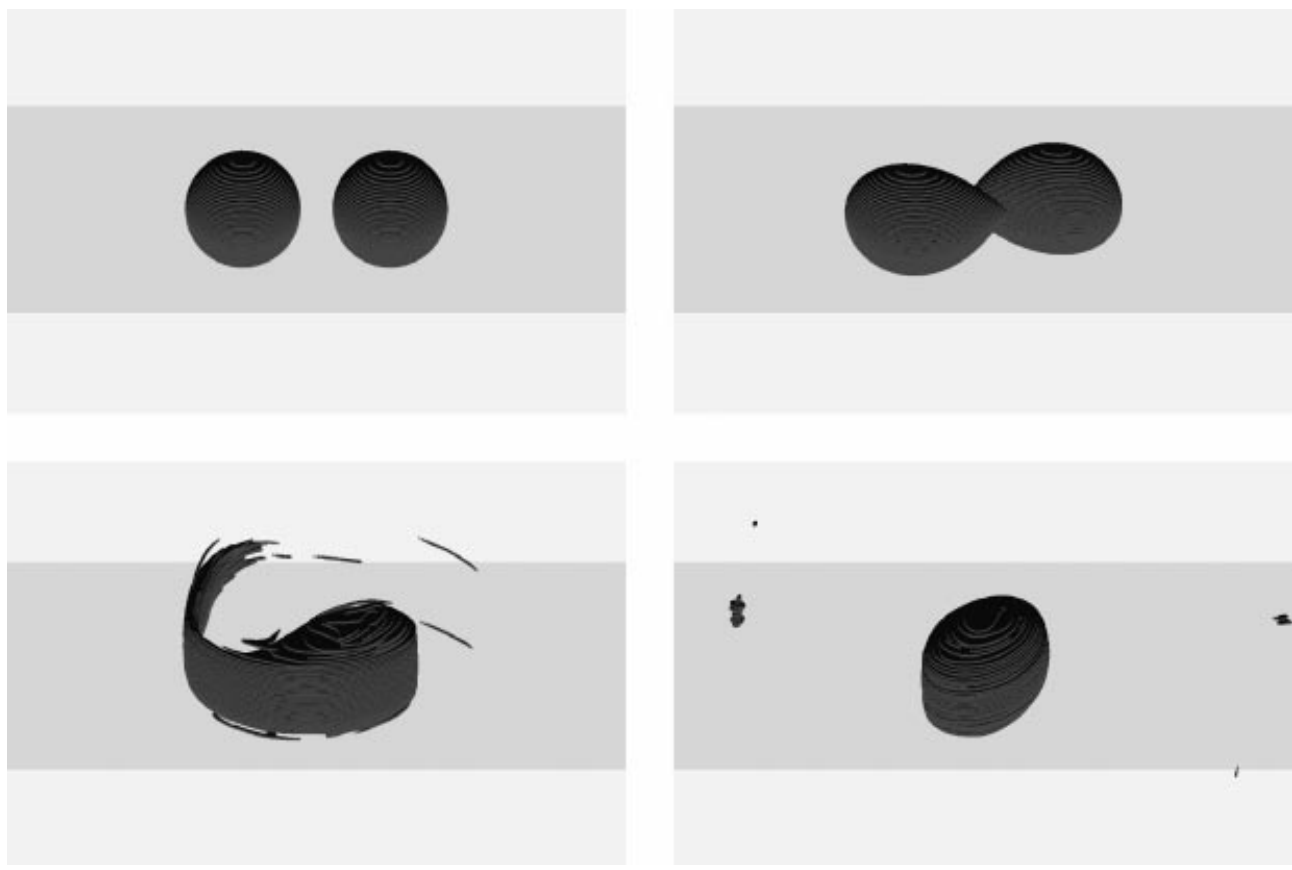

FIGURE 16. CM: time evolution left to right, top to bottom $(t=0,1,11,100)$ with $\delta z / R=0$, $\delta x / R=2.55 . \rho_{V}=0.0022$ at $t=100$.

contours making up each identifiable vortex, we calculate the vortex volume $V$ and the height-to-width aspect ratio $h / r$. Here, $h$ is the half-height of the vortex and $r$ is the mean horizontal radius, defined implicitly by $V=(4 \pi / 3) h r^{2}$.

The possible interactions between the two vortices fall into four different regimes, depending on the initial horizontal and vertical offsets. These regimes are defined in Dritschel \& Waugh (1992) in two-dimensional flows and in Dritschel (2002) for the three-dimensional case. The first is 'elastic interaction' (hereinafter referred to as EI) and corresponds to an interaction where the vortices never touch (this occurs for well-separated vortices). In the other cases where the two vortices first merge and possibly create secondary vortices, we define the relative PV volume ratio $\rho_{V}$ :

$$
\rho_{V}=\frac{V_{t o t}-V_{\max }}{V_{\max }},
$$

where $V_{\text {tot }}$ is the total volume of PV present in the flow and $V_{\max }$ is the volume of the biggest vortex. For $\rho_{V} \geqslant 0.9$, the interaction is called 'weak exchange' or WE. In this regime, vortices first merge, exchange only a small amount of PV and then eventually separate. For $0.1 \leqslant \rho_{V} \leqslant 0.9$, the interaction is called 'partial merger' or PM. Finally for $\rho_{V} \leqslant 0.1$ the interaction is called 'complete merger' or CM.

\subsection{Results}

We first illustrate these interaction regimes with four examples in which the two vortices are horizontally aligned. The results are presented in figures $16,17,18$ and 19. The CM regime is illustrated in figure 16 , for a horizontal separation $\delta x / R=2.55$. The two vortices merge rapidly by $t \simeq 1$ and from $t \geqslant 4$ create a small amount of debris. The volume of the final main vortex is $85.8 \%$ of the initial total volume of $\mathrm{PV}$, representing a $70 \%$ growth in volume. Note that the height of the main vortex 


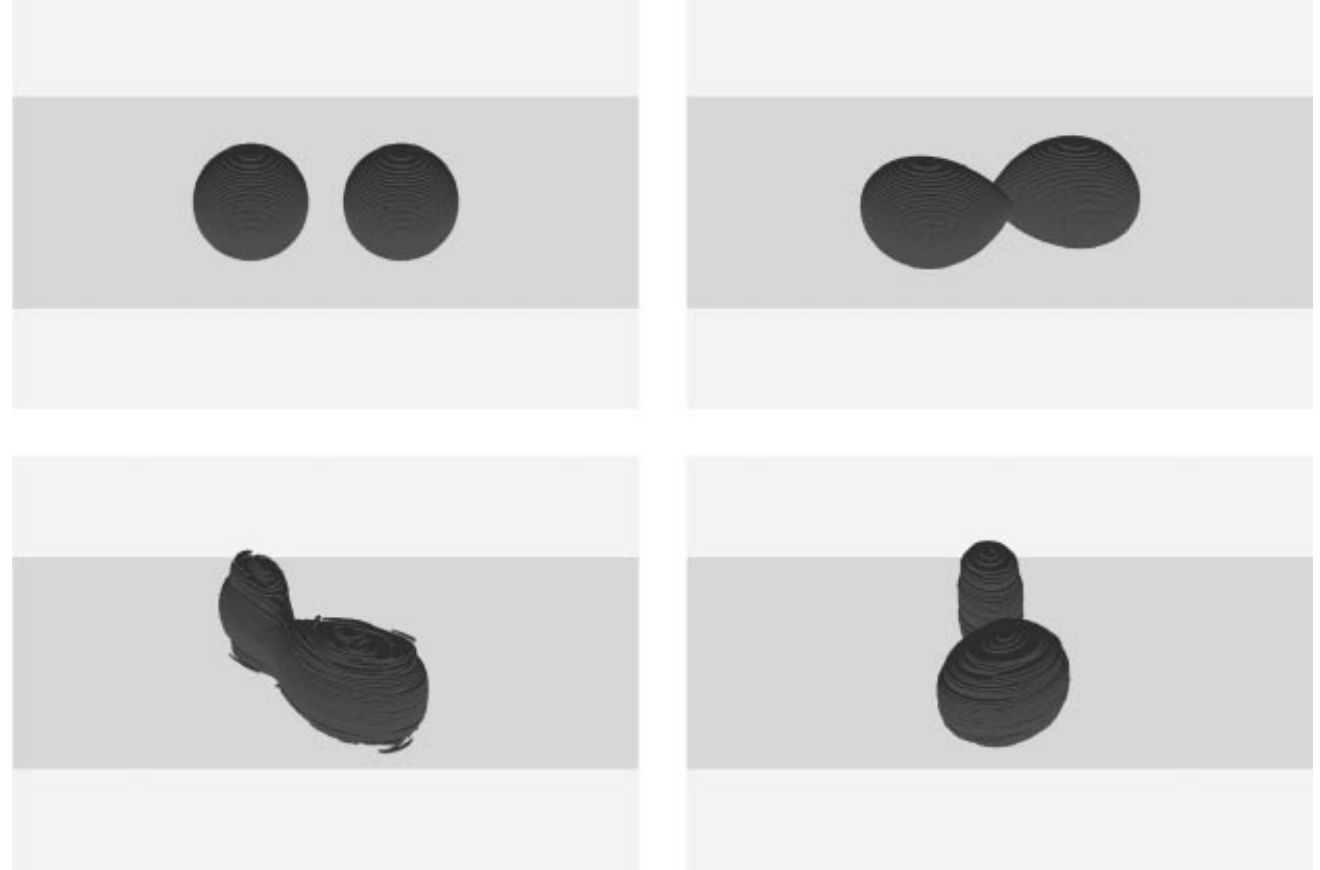

FIGURE 17. PM: time evolution $(t=0,1,11,100)$ with $\delta z / R=0, \delta x / R=2.6$. $\rho_{V}=0.4926$ at $t=100$.

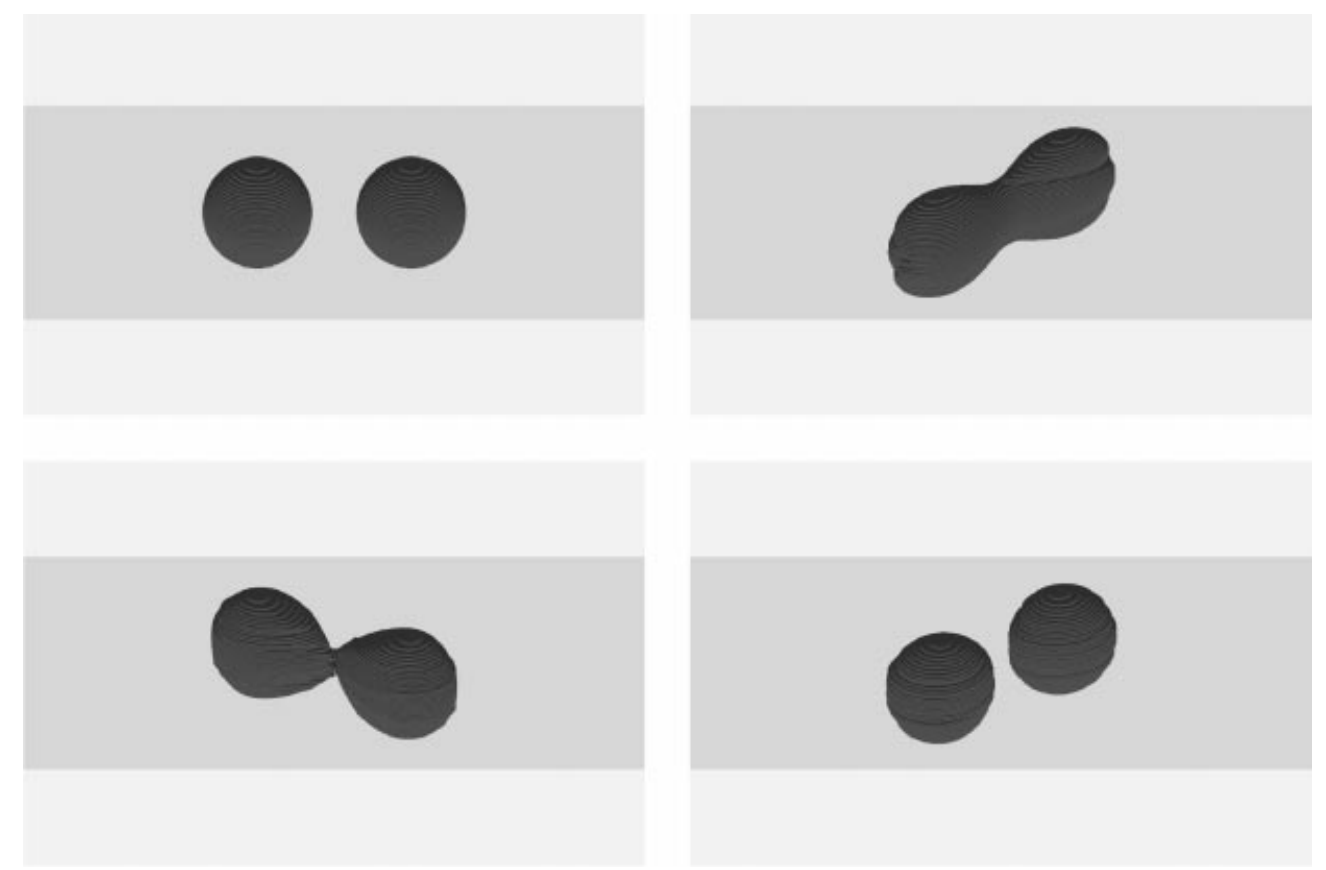

FigURE 18. WE: time evolution $(t=0,1.5,5,100)$ with $\delta z / R=0, \delta x / R=2.8$. $\rho_{V}=0.9905$ at $t=100$. 

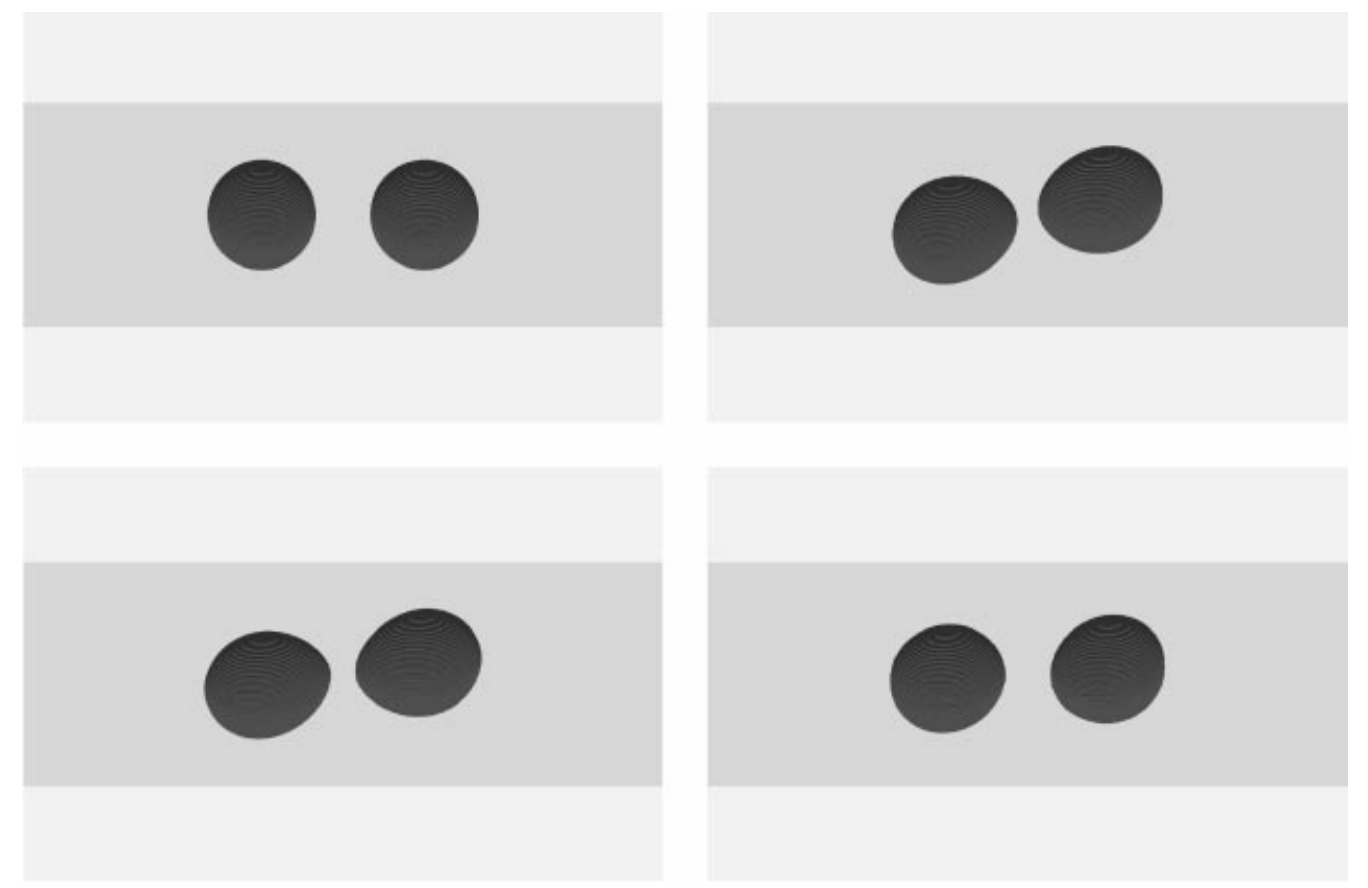

Figure 19. EI: time evolution $(t=0,1,9,100)$ with $\delta z / R=0, \delta x / R=3.0 . \rho_{V}=1$.

remains unchanged. On the other hand, we have $\rho_{V}=0.0022$ at $t=100$. The PM regime is illustrated in figure 17 for $\delta x / R=2.6$. The two vortices again merge rapidly and generate fine-scale debris from $t \geqslant 5$. But here at $t=12.5$, the main vortex splits into two secondary asymmetric vortices with a volume ratio of 2.92 . The debris then rapidly thin and disperse. From $t=28.5$, the two secondary vortices occasionally generate small tertiary vortices; however, the volume of the two vortices decreases by only $0.55 \%$ from $28.5<t<100$. By $t=100$, only two vortices remain, and their combined volume is $97 \%$ of the original total volume, and $\rho_{V}=0.4926$. This interaction generates relatively little debris compared to the $\mathrm{CM}$ interaction previously illustrated. In this sense PM is a weaker interaction than CM. Note again that the vortex height does not decrease for both product vortices. The WE regime is illustrated in figure 18 for $\delta x / R=2.8$. This interaction consists of a succession of contacts between the two vortices but very little volume exchange and virtually no debris. The two vortices remain nearly symmetric and retain most of their original volume. In this case, $\rho_{V}=0.9905$ at $t=100$. Finally, the EI regime is illustrated in figure 19 , for $\delta x / R=3.0$. Here, the vortices only slightly deform quasi-periodically in time without ever touching.

We now examine a few examples with a non-zero vertical offset $\delta z$. Each example is taken just inside the CM regime, at the largest or 'critical' horizontal separation for which CM occurs. An example of moderate vertical separation $(\delta z / R=0.5$, $\delta x / R=2.5$ ) is shown in figure 20. The two vortices merge at $t \simeq 1$ and generate debris from $t \geqslant 2.5$. By $t=100$, the main vortex contains $81.1 \%$ of the initial total volume of $\mathrm{PV}$, compared to the $85.8 \%$ in the $\delta z / R=0$ case discussed above, so that here slightly more debris has been generated. In this case, $\rho_{V}=0.0007$ at $t=100$. However the phenomenology is similar. Increasing the vertical separation to $\delta z / R=1(\delta x / R=2.55)$ begins to show differences-see figure 21. Merger occurs 

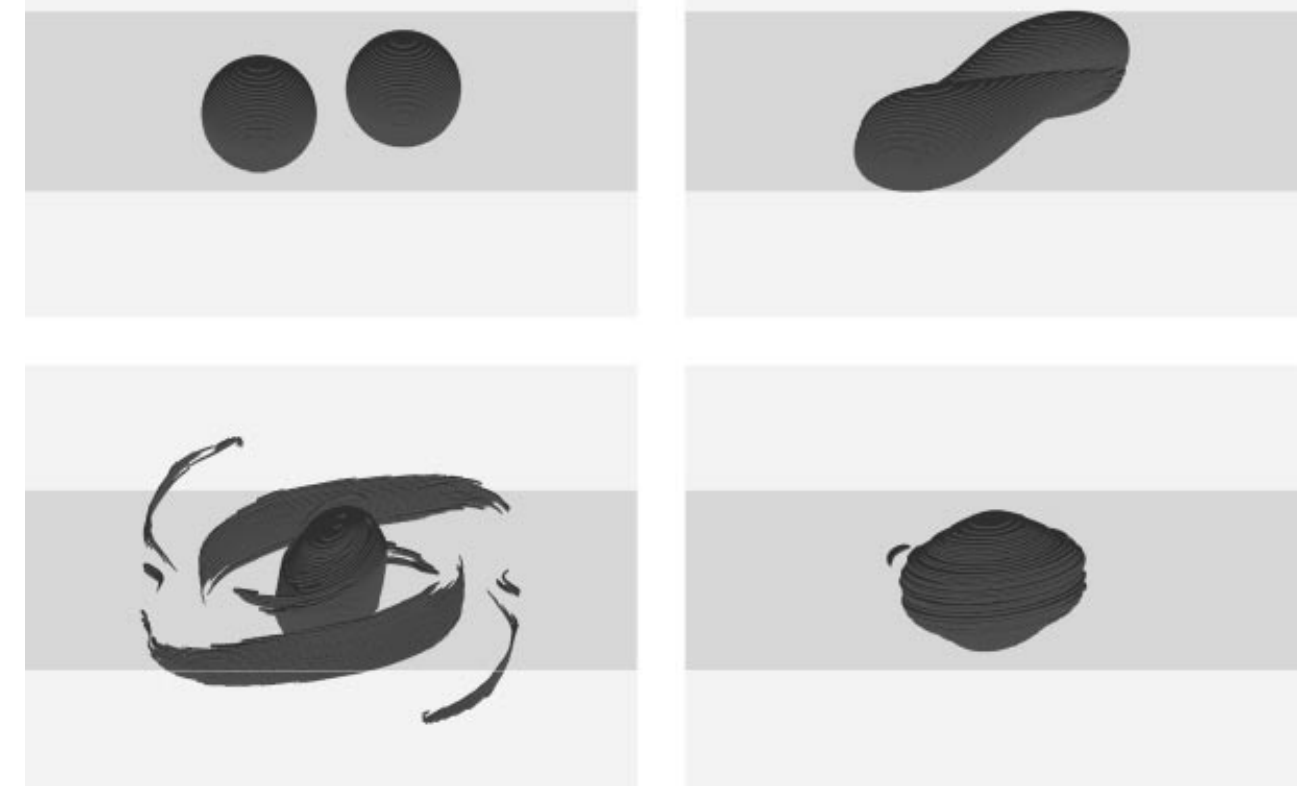

FiguRE 20. CM: time evolution $(t=0,1,7,100)$ with $\delta z / R=0.5, \delta x / R=2.5$. $\rho_{V}=0.0007$ at $t=100$.

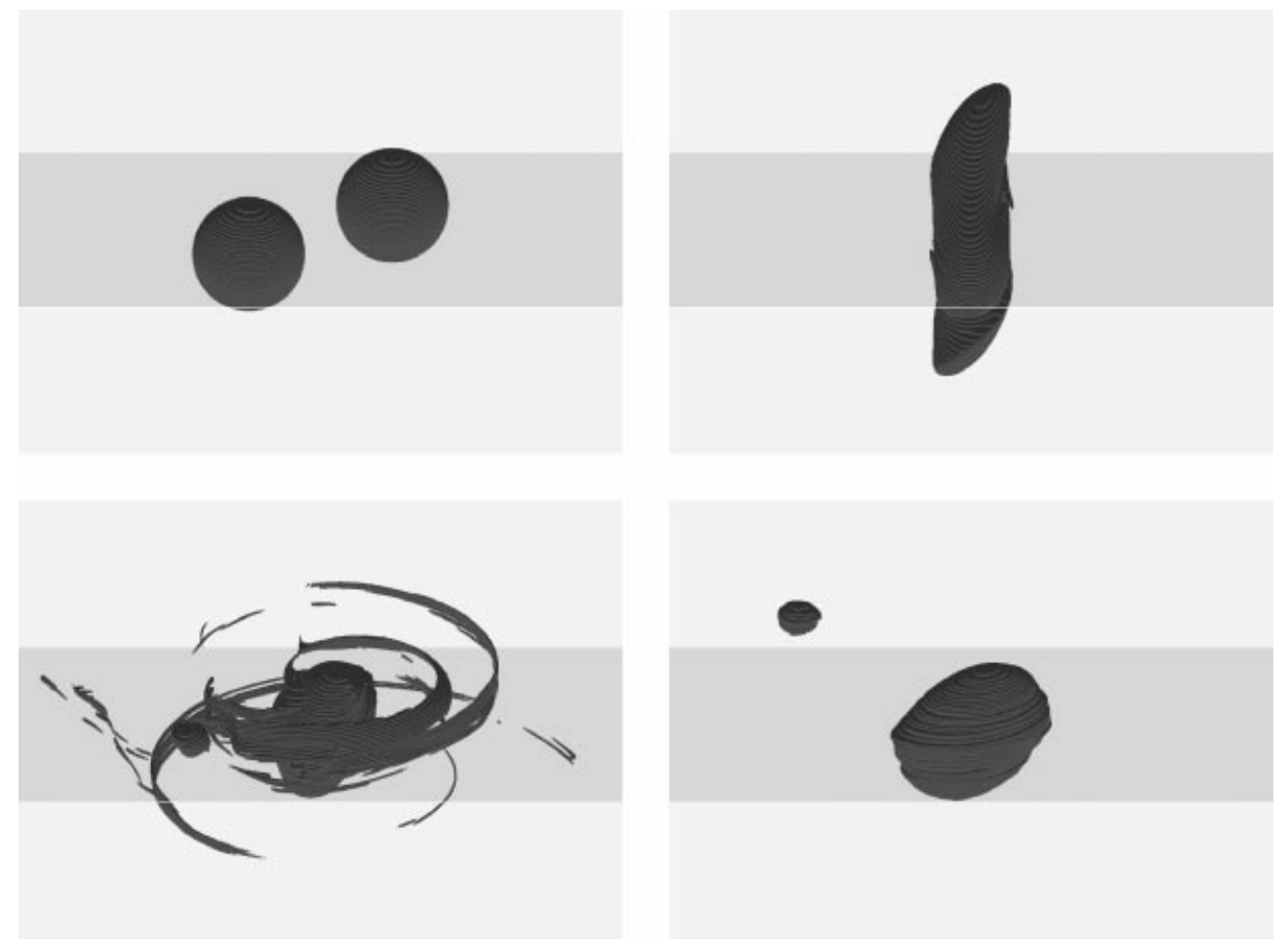

Figure 21. CM: time evolution $(t=0,2.5,17,100)$ with $\delta z / R=1.0, \delta x / R=2.55$.

$$
\rho_{V}=0.0226 \text { at } t=100 \text {. }
$$



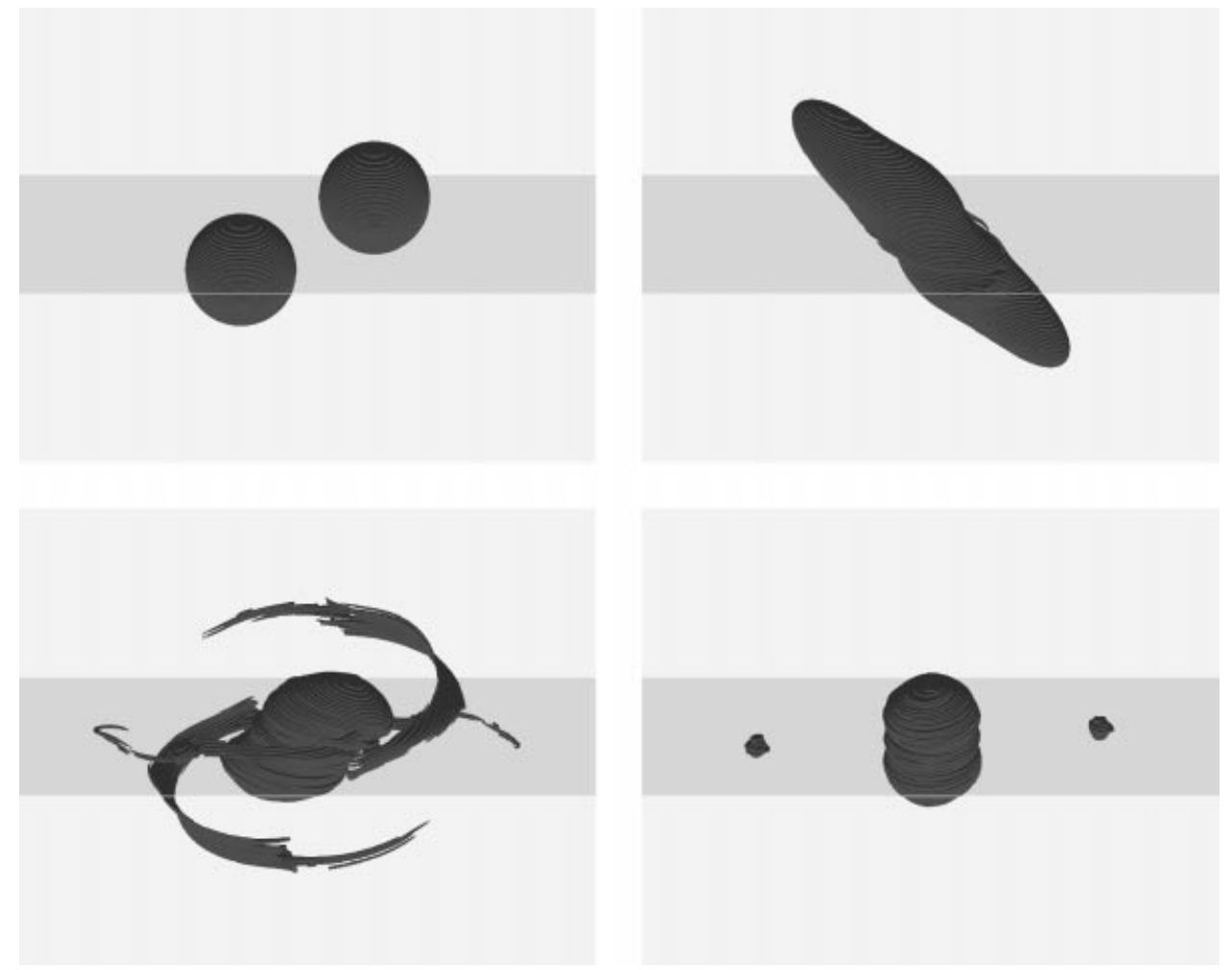

Figure 22. CM: time evolution $(t=0,4.5,13.5,100)$ with $\delta z / R=1.5, \delta x / R=2.4$.

$$
\rho_{V}=0.0087 \text { at } t=100 \text {. }
$$

at about $t \simeq 1$ and debris is generated from $t \geqslant 4.5$. The final volume of the main vortex is $78.4 \%$ of the initial volume of $\mathrm{PV}$ and $\rho_{V}=0.0226$ at $t=100$. On the other hand, the vortex no longer spans the total vertical height of the original vortices, but only $88.3 \%$ of it. Part of the original merged vortex detaches at some stage, reducing the height of the main vortex. For a greater vertical offset, $\delta z / R=1.5$ $(\delta x / R=2.4)$, as shown in figure 22 , the situation is similar as the main final vortex spans $88.6 \%$ of the original height of the two vortices. The volume of the main structure becomes $78.3 \%$ of the initial volume of $\mathrm{PV}$, and $\rho_{V}=0.0087$ at $t=100$. Finally for $\delta z / R=1.7(\delta x / R=2.25)$, the situation is again different - see figure 23 . In this case the vortices first merge at $t=1.5$. Only a negligible amount of debris is generated around $t \simeq 6.5$, and the final vortex volume approximately equals the initial total volume of PV. As a consequence, the vortex spans the total initial height spanned by the original vortices. Note too that there has been a significant change in the structure of the final vortex, from a roughly ellipsoidal shape to a dumbbell shape.

The location of the four different interaction regimes (EI, WE, PM and CM) in the parameter plane $(\delta x / R, \delta z / R)$ is now examined. The results of all simulations are summarized in figure 24 . The circular arc of radius $2 R$ shown in the figure gives the limit below which the two vortices initially overlap. It should be noted that for initially spherical vortices the relative horizontal gap $\delta / R$ reduces to $\delta x / R-2$ while for the steady states $\delta x / R$ was found to be a function of the vertical offset (cf. figure 7). Here $\delta$ and $\delta x$ are equivalent measures of the initial horizontal separation between the vortices. 

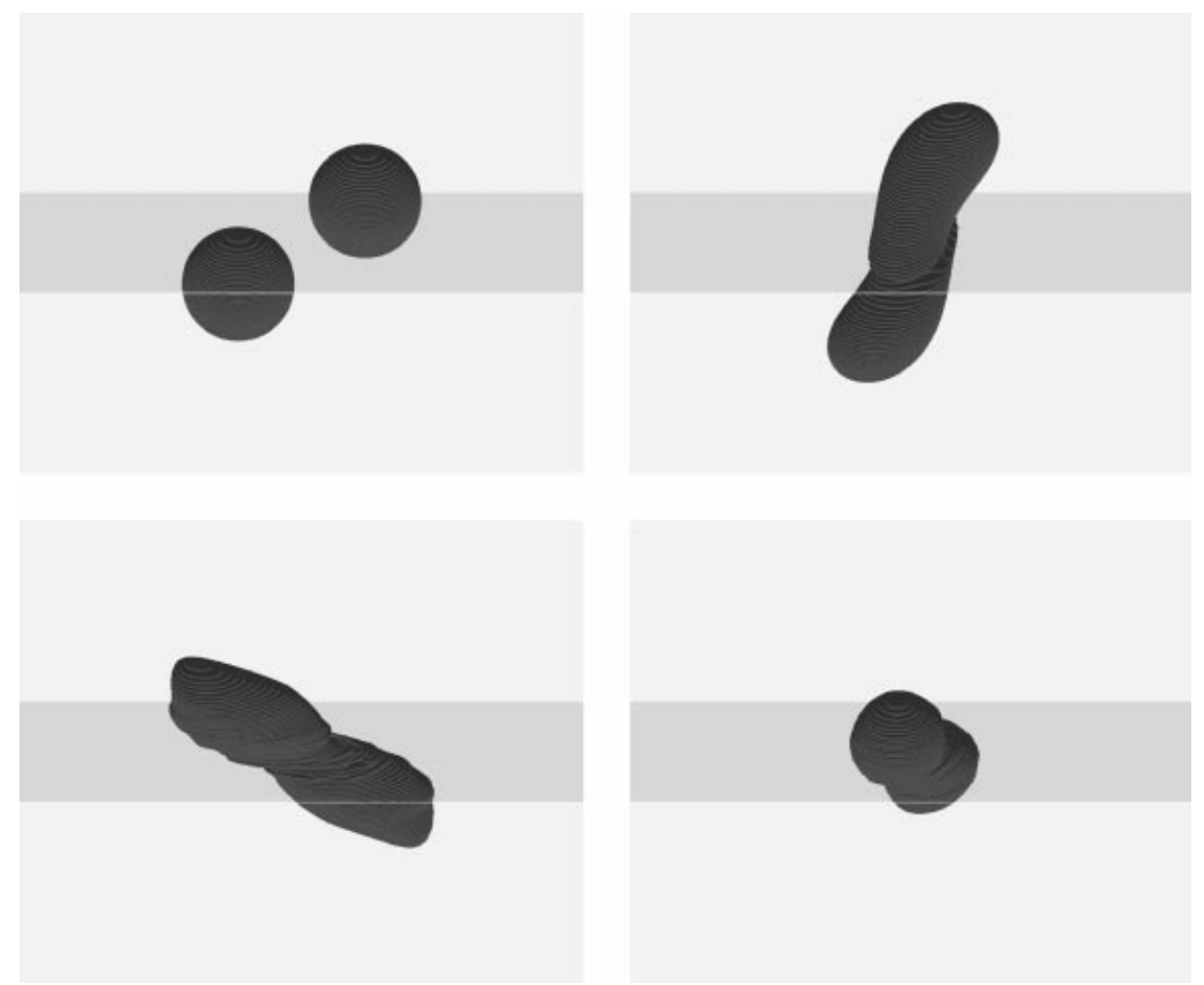

Figure 23. CM: time evolution $(t=0,2.5,18,100)$ with $\delta z / R=1.7, \delta x / R=2.25 . \rho_{V}=0$.

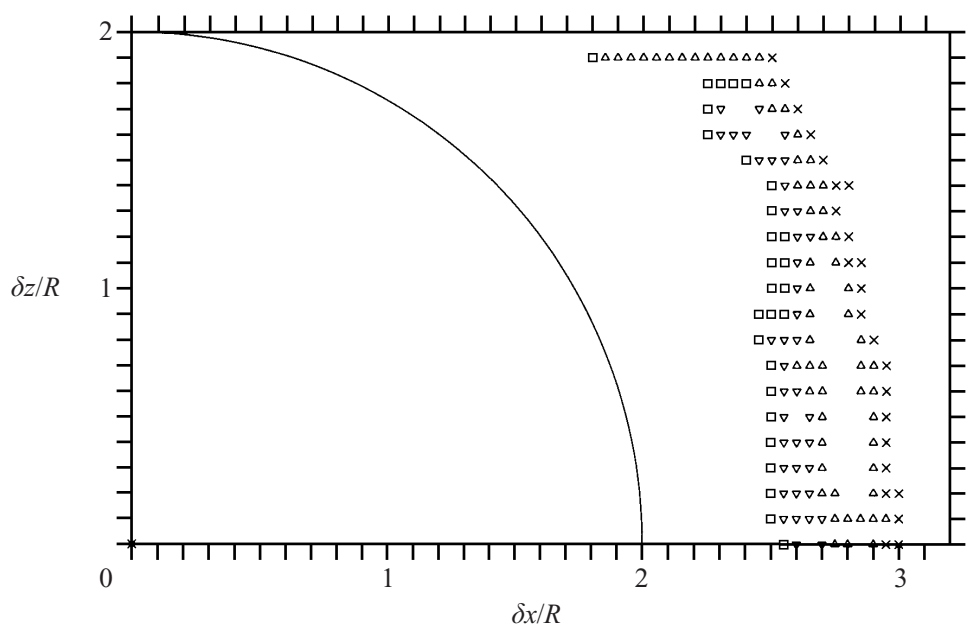

FIGURE 24. Critical merger offsets as estimated from the nonlinear simulations. The squares indicate $\mathrm{CM}$, inverted triangles PM, triangles WE and crosses EI. The circular arc of radius 2 indicates the contact curve.

It is noteworthy that the curve dividing the $\mathrm{CM}$ and PM regimes is qualitatively similar in shape to the marginal stability curve for the steady states presented in figure 14. It is seen that $\delta x / R$ exhibits a local minimum around $\delta z / R \sim 1$ along this curve. This confirms that such a vertical separation is most favourable for a 


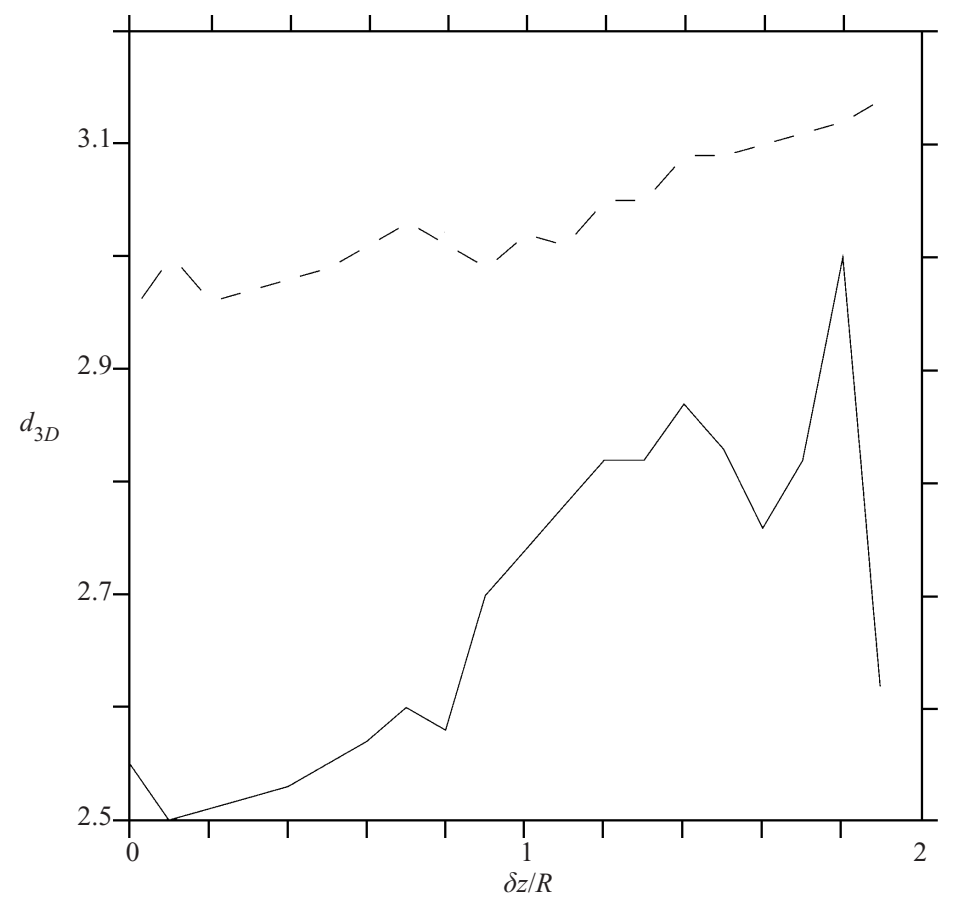

FIGURE 25. Critical distances $d_{3 D, C M} / R$ (solid line) and $d_{3 D, W E} / R$ (dashed line) for the CASL simulations versus $\delta z / R$.

strong interaction. On the other hand, the limit curve for the EI regime has $\delta x / R$ monotonically decreasing with $\delta z / R$. Note that the PM regime disappears for $\delta z / R$ between 1.7 and 1.8. This coincides with the formation of dumbbell vortices in the $\mathrm{CM}$ regime for $\delta z / R>1.7$. The bulge in the width of the PM regime seen just below $\delta z / R=1.7$ represents interactions which produce temporary dumbbell vortices which subsequently divide asymmetrically. This asymmetry decreases as $\delta z / R$ increases, and eventually a stable, nearly symmetric dumbbell vortex is produced $(\mathrm{CM})$.

Finally, for large vertical offsets $(\delta z / R>1.8)$ the horizontal separation required for CM strongly decreases, while the WE regime exists for a large range of $\delta x / R$.

The normalized three-dimensional distance below which $C M$ and WE occur, $d_{3 D, C M} / R$ and $d_{3 D, W E} / R$ respectively, are plotted as functions of $\delta z / R$ in figure 25. It is seen that $d_{3 D, C M} / R$ generally increases with $\delta z$ even for large vertical offsets, except for the final sudden collapse when $\delta z / R>1.8$. On the other hand $d_{3 D, W E} / R$ increases (less steeply) with $\delta z$ even for $\delta z / R$ close to 2, beyond which only EI is possible. These tendencies, at least in the range $0<\delta z / R<1.44$ agree qualitatively with the linear stability findings presented in $\S 4$ (cf. figure 12).

We next examine the properties of the final main vortex for the largest $\delta x / R$ in the $\mathrm{CM}$ regime, as a function of the relative vertical offset $\delta z / R$. We focus attention on the volume $V$ and the height-to-width aspect ratio $h / r$ of the vortex. For comparison, note that perfect merger with no debris would result in identical initial and final total volume and an aspect ratio of

$$
h / r=(h / r)_{\text {ideal }}=\frac{1}{\sqrt{2}}\left(1+\frac{\delta z}{2 R}\right)^{3 / 2} .
$$




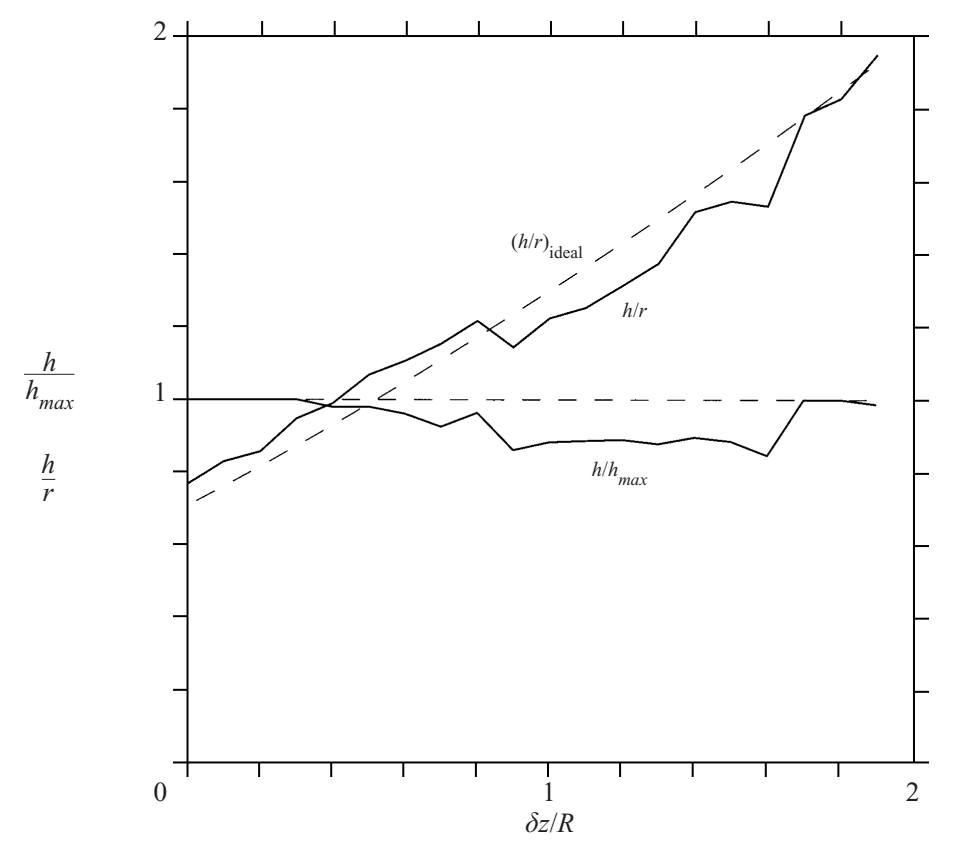

FIGURE 26. Height-to-width aspect ratio $h / r$ of the first CM versus $\delta z$ and the ratio of the half-height $h$ to the maximum one $h_{\max }$. $h_{\text {ideal }}$ is also plotted for comparision.

We recall that $r$ is related to the volume and the height of the vortex by $V=(4 \pi / 3) h r^{2}$. In figure 26, we show both the ratio between the actual half-height $h$ of the vortex and the maximum possible one $\left(h_{\max }=R+\delta z / 2\right)$ and the aspect ratio $h / r ;(h / r)_{\text {ideal }}$ is also plotted for comparison (dashed line). For $\delta z / R \leqslant 0.8$, the actual aspect ratio of the main vortex is greater than $(h / r)_{\text {ideal }}$ and $h \simeq h_{\max }$. This is a consequence of the formation of lateral debris that reduces the mean horizontal radius $r$ of the vortex, increasing $h / r$. For $0.8<\delta z / R<1.7$, the ratio $h / r$ is less than $(h / r)_{\text {ideal }}$ and $h<h_{\max }$. In this range, the nonlinear evolution tends to limit the aspect ratio by ejecting PV from the vertical extremities of the initially formed compound vortex. Similar results are found in Dritschel (2002) for tall, horizontally aligned vortices. This behaviour reduces $h / r$ for all vortices, consistent with the 'tall column' instability described in Dritschel \& de la Torre Juárez (1996). Finally, for $\delta z / R>1.7, h / r \simeq(h / r)_{\text {ideal }}$ and $h \simeq$ $h_{\max }$. Here a dumbbell vortex is formed with little debris. However we emphasize, as seen from figure 24 for $\delta z / R=1.9$, that the compound vortex may be meta-stable - the interaction often ends as a WE because the vortex eventually separates (cf. figure 9).

The ratio of final to initial total volume of PV is shown in figure 27. This also indicates a distinct change in the nature of the interaction for $\delta z / R \geqslant 1.7$. For such vertical offsets, the vortex volume ratio is nearly unity. For $\delta z / R \leqslant 1.7$ the mean volume ratio is approximately 0.8 , which means that the typical interaction of this type leads to a $60 \%$ increase in the vortex volume. However, this sort of increase is not typical of the PM and WE regimes, which arguably are more likely to be encountered in flows with many interacting vortices.

\section{Conclusion}

In this study, we have found the equilibrium shapes of two identical uniform-PV quasi-geostrophic vortices, offset both horizontally and vertically. Next, we determined 


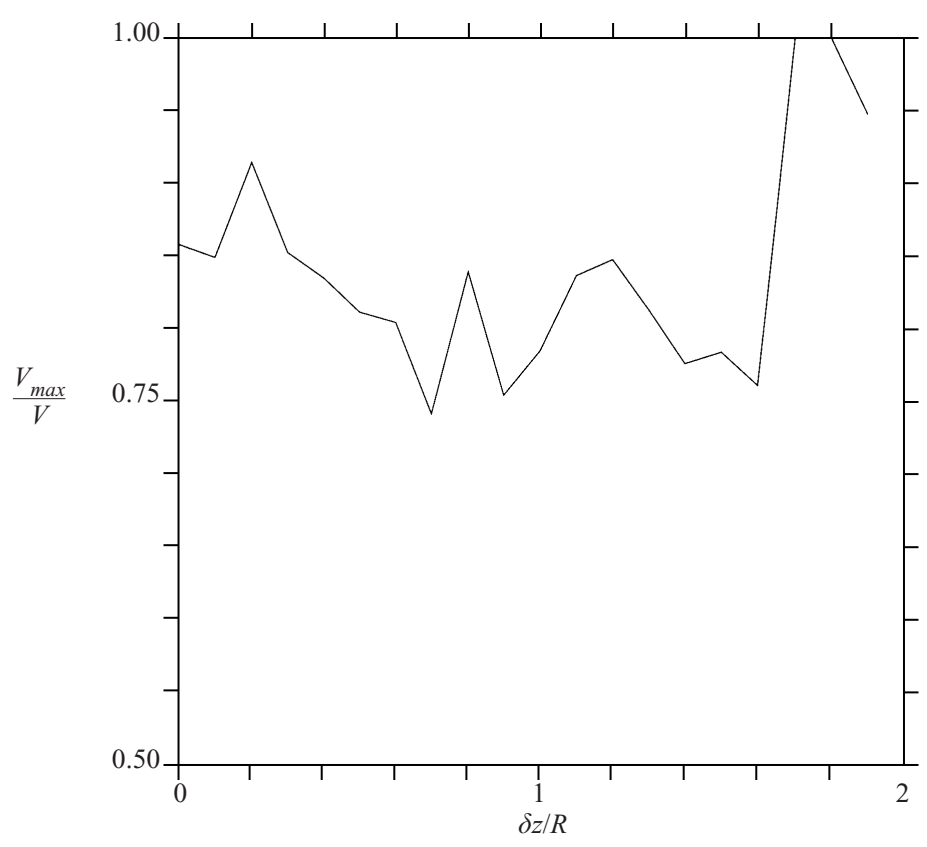

FIGURE 27. Volume ratio $\left(V_{\max } / V\right)$ for the first $\mathrm{CM}$ versus $\delta z$.

their linear stability, and finally we examined the nonlinear interaction of spherical vortices, roughly approximating the equilibrium states.

The equilibrium shapes exhibit a perhaps surprising trend. Normally, as the threedimensional separation distance between vortices increases, one would expect the deformation of each vortex to decrease, since the mean straining of one vortex on the other decreases. However, we have found that this deformation increases at first when we hold the horizontal offset constant and increase the vertical offset from zero. Maximum deformation occurs when the vertical offset is comparable to the mean vortex radius.

The linear stability results furthermore indicate that vortices offset moderately in the vertical destabilize sooner (with increasing horizontal offset) than do horizontally aligned vortices. As a function of the vertical offset, the critical horizontal offset for instability increases only weakly, but it increases nonetheless, and the implication is that vortices destabilize from greater overall separation distances when they are offset moderately in the vertical. Evidently, the deformation of the vortex is the most important factor affecting stability. The nonlinear results furthermore support this finding: moderately vertically offset vortices merge at slightly greater horizontal offsets than do horizontally aligned vortices.

What could be the explanation for this? We hypothesize that the observed behaviour is fundamentally due to the anisotropy of the quasi-geostrophic equations, in particular the lack of vertical motion. This anisotropy was examined in Reinaud et al. (2003), who analysed the results from a number of high-resolution simulations of quasi-geostrophic turbulence, and who also developed a simple model for the mean shape (height-to-width aspect ratio) of a vortex in a random background straining flow. It was found that vortices exhibit a mean aspect ratio of 0.8 (after scaling on $f / N$ ), this value being the result of both a detailed statistical analysis and the simple model. The simple model considered a single ellipsoidal vortex in a linear background 
flow (McKiver \& Dritschel 2003), approximating the leading-order effects of another vortex arbitrarily positioned in space. In such a linear flow, the ellipsoid remains an ellipsoid, but in general deforms. Reinaud et al. (2003) found that an ellipsoid with a mean aspect ratio of 0.8 is best able to survive in this background flow, or is least likely to be torn apart (be extended indefinitely).

If the quasi-geostrophic equations were isotropic, then vortices would exhibit a mean aspect ratio of 1 . Anisotropy - the lack of vertical advection - is responsible for the lower observed value. Anisotropy appears as a difference between the effects of vertical shear (the vertical gradient of the horizontal flow) and the effects of horizontal shear (the horizontal gradient of the horizontal flow). Vertical shear tilts a vortex, increasing its overall extension, and in particular increases the three-dimensional distance between its vertical extremities. Horizontal shear squashes the vortex horizontally, and instead increases the distance between its horizontal extremities. In general, both vertical and horizontal shear are present. The unit aspect ratio vortices considered in the present study have a larger aspect ratio than the observed mean value of 0.8 , and this implies that they are more sensitive to the effects of vertical shear, which arises once the vortices are offset vertically. Generally, such vortices are more easily deformed than vortices having an aspect ratio of 0.8 .

It would be worth considering the interaction between vortices of the observed mean aspect ratio, 0.8 -in this case we conjecture that vortex deformation will decrease monotonically with increased three-dimensional separation. Vertical shear will then be less able to deform the vortices.

A complete understanding of vortex interactions, however, will require a much broader investigation which additionally considers different vortex volumes, different aspect ratios, etc. A direct attack on this problem appears impossible at present, due to the high cost of finding equilibrium states and performing nonlinear simulations. However, an indirect attack, based on an extension of the ellipsoidal model described above to several interacting vortices, is at present under development and may enable a comprehensive, qualitative understanding of vortex interactions in a quasi-geostrophic fluid.

J.N.R. is supported by the UK EPSRC (Grant GR/N11711).

\section{Appendix}

The principle of the method is to find the shapes of the contours bounding the vortex in each horizontal cross-section which coincide with isolines of the streamfunction $\psi$, in the relevant rotating reference frame. In this reference frame, the velocity normal to the boundary is zero, and therefore the contours do not deform in time. Here, each vortex is represented by a collection of horizontal layers of uniform PV. Each vortex boundary is thus composed of a set of contours $\mathscr{C}_{k}$ and their associated vertical thickness $\Delta z$. The thickness is here taken to be the same for every layer although this choice is not necessary in the method.

The streamfunction in the rotating frame $\tilde{\psi}_{k, n}$ along the $k$ th contour $\left(\mathscr{C}_{k}\right)$ in the $n$th iteration - or $n$th guess for the vortex shape-takes the form

$$
\tilde{\psi}_{k, n}\left(\boldsymbol{\rho}_{k, n}\right)=\psi_{k, n}\left(\boldsymbol{\rho}_{k, n}\right)-\frac{1}{2} \Omega_{n} \boldsymbol{\rho}_{k, n}^{2},
$$

where $\boldsymbol{\rho}_{k, n}=\left(x_{k, n}, y_{k, n}\right)$ is the horizontal position vector describing the boundary of the contour $\mathscr{C}_{k}$ while $\Omega_{n}$ is the $n$th estimate for the background rotation, and $\psi_{k, n}\left(\boldsymbol{\rho}_{k, n}\right)$ is the streamfunction obtained from the inversion of Poisson's equation (2). 
For the $(n+1)$ th iteration, we enforce, approximately, the condition of equilibrium, namely

$$
\tilde{\psi}_{k, n+1}\left(\boldsymbol{\rho}_{k, n+1}\right)=c_{k},
$$

where $c_{k}$ is a constant (generally different for each contour $\mathscr{C}_{k}$ ). Starting from the $n$th guess for the equilibrium solution, this equation is partially linearized about $\boldsymbol{\rho}_{k, n}$ to find the correction $\boldsymbol{\rho}_{k, n+1}-\boldsymbol{\rho}_{k, n}$. We use here radial corrections, where the radius is measured horizontally from the centroid of the vortex to which $\mathscr{C}_{k}$ belongs. We write

$$
\boldsymbol{\rho}_{k, n+1}=\boldsymbol{\rho}_{k, n}+\eta_{k, n} \hat{\boldsymbol{r}}_{k, n},
$$

where $\eta_{k, n}$ is the radial correction or displacement, and $\hat{\boldsymbol{r}}_{k, n}$ is the unit vector in this direction. Equation (5) is then expanded to first order in $\eta$, but ignoring the implicit change in $\psi$ associated with the change in the contour shapes:

$$
\begin{aligned}
\tilde{\psi}_{k, n+1}\left(\boldsymbol{\rho}_{k, n+1}\right) \simeq & \psi_{k, n}\left(\boldsymbol{\rho}_{k, n}\right)-\frac{1}{2} \Omega_{n} \boldsymbol{\rho}_{k, n}^{2}-\frac{1}{2} \Omega^{\prime} \boldsymbol{\rho}_{k, n}^{2} \\
& +\eta_{k, n}\left[\left(\hat{\boldsymbol{r}}_{k, n} \cdot \nabla\right) \psi_{k, n}\left(\boldsymbol{\rho}_{k, n}\right)-\Omega_{n} \boldsymbol{\rho}_{k, n} \cdot \boldsymbol{r}_{k, n}\right],
\end{aligned}
$$

where

$$
\Omega^{\prime}=\Omega_{n+1}-\Omega_{n}
$$

is the correction to the background rotation rate and

$$
\psi_{k, n+1} \simeq \psi_{k, n}
$$

is heuristically assumed, following Pierrehumbert (1980). The latter assumption avoids the large matrix problem that would otherwise result.

Thus, from (5) and (7), the correction $\eta_{k, n}$ can be expressed as a simple function of the set of constants $c_{k}$ and $\Omega^{\prime}$ :

$$
\eta_{k, n}=\frac{c_{k}-\tilde{\psi}_{k, n}\left(\boldsymbol{\rho}_{k, n}\right)+\frac{1}{2} \Omega^{\prime} \boldsymbol{\rho}_{k, n}^{2}}{\xi_{k, n}},
$$

where

$$
\xi_{k, n}=\left(\hat{\boldsymbol{r}}_{k, n} \cdot \nabla\right) \psi_{k, n}\left(\boldsymbol{\rho}_{k, n}\right)-\Omega_{n} \boldsymbol{\rho}_{k, n} \cdot \hat{\boldsymbol{r}}_{k, n}
$$

is the azimuthal component of the velocity.

Two other conditions are now imposed to determine the rotation rate correction $\Omega^{\prime}$ and the constants $c_{k}$. The first one is the volume conservation of the vortices. In the absence of vertical mass transfer in QG flows, volume conservation is equivalent to the conservation of the area $\mathscr{A}_{k}$ within each contour $\mathscr{C}_{k}$. This can be expressed between two iterations, at the first order, by

$$
A_{k, n+1}=\frac{1}{2} \oint_{\mathscr{C}_{k}} \boldsymbol{\rho}_{k, n+1}^{2} \mathrm{~d} \theta \simeq A_{k, n}+\oint_{\mathscr{C}_{k}} \eta_{k, n} \hat{\boldsymbol{r}}_{k, n} \cdot \boldsymbol{\rho}_{k, n} \mathrm{~d} \theta \equiv \mathscr{A}_{k},
$$

where $\theta$ is the geometric polar angle, $A_{k, n}$ is the area of the $k$ th contour at the $n$th iteration, and $\mathscr{A}_{k}$ is the prescribed area of $\mathscr{C}_{k}$ (note that $A_{k, n}$ converges to $\mathscr{A}_{k}$ as $\eta_{k, n} \rightarrow 0$ ). Substituting $\eta_{k, n}$ from (A 7), we obtain the following equations for the constant $c_{k}$ as a function of $\Omega^{\prime}$ :

$$
c_{k}=h_{k, n}-\Omega^{\prime} g_{k, n}
$$


where (with $w_{k, n} \equiv \xi_{k, n}^{-1} \boldsymbol{r}_{k, n} \cdot \boldsymbol{\rho}_{k, n}$ )

$$
h_{k, n}=\frac{\mathscr{A}_{k}-A_{k, n}+\oint_{\mathscr{C}_{k}} \tilde{\psi}_{k, n} w_{k, n} \mathrm{~d} \theta}{\oint_{\mathscr{C}_{k}} w_{k, n} \mathrm{~d} \theta} \text { and } g_{k, n}=\frac{1}{2} \frac{\oint_{\mathscr{C}_{k}} \boldsymbol{\rho}_{k, n}^{2} w_{k, n} \mathrm{~d} \theta}{\oint_{\mathscr{C}_{k}} w_{k, n} \mathrm{~d} \theta} \text {. }
$$

A second constraint is now imposed to determine the rotation rate correction $\Omega^{\prime}$. We fix the distance between two given points chosen from the two vortices. This implies that the sum of the local corrections at these points must vanish:

$$
\eta_{k_{1}, n}\left(\theta_{1}\right)+\eta_{k_{2}, n}\left(\theta_{2}\right)=0 .
$$

Here $k_{1}$ and $k_{2}$ refer to the mid-contour of each vortex and the two points (with polar angle $\theta_{1}$ and $\theta_{2}$ ) lie along the innermost edges of these contours (see figure 1). Using equation (A 7) again, we find

$$
\Omega^{\prime}=\frac{\frac{1}{\xi_{k_{1}, n}\left(\theta_{1}\right)}\left[\tilde{\psi}_{k_{1}, n}\left(\theta_{1}\right)-h_{k_{1}, n}\right]+\frac{1}{\xi_{k_{2}, n}\left(\theta_{2}\right)}\left[\tilde{\psi}_{k_{2}, n}\left(\theta_{2}\right)-h_{k_{2}, n}\right]}{\frac{1}{\xi_{k_{1}, n}\left(\theta_{1}\right)}\left[\frac{1}{2} \boldsymbol{\rho}_{k_{1}, n}^{2}\left(\theta_{1}\right)-g_{k_{1}, n}\right]+\frac{1}{\xi_{k_{2}, n}\left(\theta_{2}\right)}\left[\frac{1}{2} \boldsymbol{\rho}_{k_{2}, n}^{2}\left(\theta_{2}\right)-g_{k_{2}, n}\right]} .
$$

The streamfunction $\psi_{k, n}$ is obtained from the PV distribution by contour integration around the $\mathscr{C}_{k}$ (see Dritschel 2002, Appendix A). The integrations, including those in (A 3), are performed using two-point Gaussian quadrature between contour nodes to reach high accuracy. Local cubic splines are used to represent $\mathscr{C}_{k}$ between contour nodes. The iterative scheme is repeated until the correction $\Omega^{\prime}$ is less than a given tolerance, namely $10^{-9}$ in the present study.

\section{REFERENCES}

Cottet, G. H. 1996 Artificial viscosity models for vortex and particle methods. J. Comput. Phys. 127, 299-308.

Dritschel, D. G. 1985 The stability and energetics of corotating uniform vortices. J. Fluid Mech. 157, 95-13.

Dritschel, D. G. 1990 The stability of elliptical vortices in an external straining flow. J. Fluid Mech. 210, 223-261.

Dritschel, D. G. 1995 A general theory for two-dimensional vortex interactions. J. Fluid Mech. 293, 269-303.

Dritschel, D. G. 2002 Vortex merger in rotating stratified flows. J. Fluid Mech. 455, 83-101.

Dritschel, D. G. \& Ambaum, M. H. P. 1997 A contour-advective semi-lagrangian algorithm for the simulation of fine-scale conservative fields. Q. J. R. Met. Soc. 123, 1097-1130.

Dritschel, D. G. \& Macaskill, C. 2000 The role of boundary conditions in the simulation of rotating, stratified turbulence. Geophys. Astrophys. Fluid Dyn. 92, 233-253.

Dritschel, D. G. \& DE LA TORRE JuÁREZ, M. 1996 The instability and breakdown of tall columnar vortices in a quasi-geostrophic fluid. J. Fluid Mech. 328, 129-160.

Dritschel, D. G., De la Torre Juárez, M. \& Ambaum, M. H. P. 1999 The three-dimensional vortical nature of atmospheric and oceanic turbulent flows. Phys. Fluids 11, 1512-1520.

Dritschel, D. G. \& Waugh, D. W. 1992 Qualification of the inelastic interaction of unequal vortices in two-dimensional vortex dynamics. Phys. Fluids A 4, 1737-1744.

Gill, A. E. 1982 Atmosphere-Ocean Dynamics. Academic.

von Hardenberg, J., McWilliams, J. C., Provenzale, A., Shchpetkin, A. \& Weiss, J. B. 2000 Vortex merging in quasi-geostrohic flows. J. Fluid Mech. 412, 331-353.

Koudella, C. R., Dritschel, D. G. \& McMullan, G. 2002 A parallel contour-advective semi- 
lagrangian algorithm for the integration of the quasi-geostrophic fluid model. J. Comput. Phys. submitted (http://www-vortex.mcs.st-and.ac.uk/ jean/kdm02.pdf).

McKiver, W. \& Dritschel, D. G. 2003 The motion of a fluid ellipsoid in a general linear background flow. J. Fluid Mech. (in press).

McWilliams, J. C. 1990 The vortices of geostrophic turbulence. J. Fluid Mech. 219, 387-404.

Pierrehumbert, R. T. 1980 A family of steady, translating vortex pairs with distributed vorticity. J. Fluid Mech. 99, 129-144.

Polvani, L. M. \& Dritschel, D. G. 1993 Wave and vortex dynamics on the surface of a sphere. J. Fluid Mech. 255, 35-64.

Polvani, L. M., Zabusky, N. J. \& Flierl, J. 1989 Two-layer geostrophic vortex dynamics. Part 1. Upper-layer V-states and merger. J. Fluid Mech. 205, 215-242.

Reinaud, J., Dritschel, D. G. \& Koudella, C. R. 2003 The shape of vortices in quasi-geostrophic turbulence. J. Fluid Mech. (in press).

Verron, J., Hopfinger, E. J. \& McWilliams, J. C. 1990 Sensitivity to initial conditions in the merging of two-layer baroclinic vortices. Phys. Fluids A 2, 886-889.

Waugh, D. W. 1992 The efficiency of symmetric vortex merger. Phys. Fluids A 4, 1745-1758. 